\title{
Pan-cancer analysis of whole genomes identifies driver rearrangements promoted by LINE-1 retrotransposition
}

\author{
Bernardo Rodriguez-Martin ${ }^{1,2,3}$, Eva G. Alvarez ${ }^{1,2,3,920}$, Adrian Baez-Ortega ${ }^{4,920}$, Jorge Zamora ${ }^{1,3,5,6,920}$, \\ Fran Supek ${ }^{7,8,920}$, Jonas Demeulemeester ${ }^{9,10}{ }^{9}$, Martin Santamarina1,2,11, Young Seok Ju ${ }^{12,13}$, \\ Javier Temes ${ }^{1,3}$, Daniel Garcia-Souto ${ }^{11}$, Harald Detering ${ }^{2,14,15}$, Yilong Li6 , Jorge Rodriguez-Castro", \\ Ana Dueso-Barroso ${ }^{16,17}$, Alicia L. Bruzos ${ }^{1,2,11}$, Stefan C. Dentro ${ }^{9,18,19}$, Miguel G. Blanco ${ }^{20,21}$, \\ Gianmarco Contino ${ }^{22}$, Daniel Ardeljan ${ }^{23}$, Marta Tojo5,14, Nicola D. Roberts ${ }^{6}{ }^{6}$, Sonia Zumalave ${ }^{10111}$, \\ Paul A. Edwards ${ }^{24,25}$, Joachim Weischenfeldt ${ }^{26,27,28}$, Montserrat Puiggròs ${ }^{17}$, Zechen Chong ${ }^{29,30}$, \\ Ken Chen ${ }^{31}$, Eunjung Alice Lee ${ }^{32}$, Jeremiah A. Wala ${ }^{33,34,35,36}$, Keiran M. Raine ${ }^{13}$, \\ Adam Butler ${ }^{13}$, Sebastian M. Waszak $\mathbb{1}^{26}$, Fabio C. P. Navarro ${ }^{37,38,39}$, Steven E. Schumacher ${ }^{33,34,35}$, \\ Jean Monlong ${ }^{40}$, Francesco Maura ${ }^{13,41,42}$, Niccolo Bolli ${ }^{41,42}$, Guillaume Bourque ${ }^{43}$, \\ Mark Gerstein $37,38,39$, Peter J. Park ${ }^{44,45}$, David C. Wedge ${ }^{13,18,46}$, Rameen Beroukhim ${ }^{33,35,36}$, \\ David Torrents ${ }^{8,17}$, Jan O. Korbel (10) ${ }^{26,47}$, Iñigo Martincorena ${ }^{6}$, Rebecca C. Fitzgerald (iD 22 , \\ Peter Van Loo ${ }^{9,10}$, Haig H. Kazazian ${ }^{23}$, Kathleen H. Burns ${ }^{48,49}$, PCAWG Structural Variation \\ Working Group ${ }^{50}$, Peter J. Campbell ${ }^{6,51,921 凶} \bowtie$, Jose M. C. Tubio ${ }^{1,2,3,13,921 \bowtie}$ and PCAWG Consortium ${ }^{52}$
}

About half of all cancers have somatic integrations of retrotransposons. Here, to characterize their role in oncogenesis, we analyzed the patterns and mechanisms of somatic retrotransposition in 2,954 cancer genomes from 38 histological cancer subtypes within the framework of the Pan-Cancer Analysis of Whole Genomes (PCAWG) project. We identified 19,166 somatically acquired retrotransposition events, which affected $35 \%$ of samples and spanned a range of event types. Long interspersed nuclear element (LINE-1; L1 hereafter) insertions emerged as the first most frequent type of somatic structural variation in esophageal adenocarcinoma, and the second most frequent in head-and-neck and colorectal cancers. Aberrant L1 integrations can delete megabase-scale regions of a chromosome, which sometimes leads to the removal of tumor-suppressor genes, and can induce complex translocations and large-scale duplications. Somatic retrotranspositions can also initiate breakagefusion-bridge cycles, leading to high-level amplification of oncogenes. These observations illuminate a relevant role of 22 L1 retrotransposition in remodeling the cancer genome, with potential implications for the development of human tumors.

1 retrotransposons are widespread repetitive elements in the human genome, representing $17 \%$ of the entire DNA content ${ }^{1,2}$. Using a combination of cellular enzymes and self-encoded proteins with endonuclease and reverse transcriptase activity, L1 elements copy and insert themselves at new genomic sites, in a process called retrotransposition. Most of the approximately 500,000 L1 copies in the human reference genome are truncated, inactive elements that are unable to retrotranspose. A small subset of them, around 100-150 L1 loci, remain active in the average human genome, acting as source elements, a small number of which consists of highly active copies termed hot-L1s $s^{3-5}$. These $\mathrm{L} 1$ source elements are usually transcriptionally repressed, but epigenetic changes that occur in tumors may promote their expression and allow them to retrotranspose $^{6,7}$. Somatic L1 retrotransposition usually introduces a new copy of the $3^{\prime}$ end of the L1 sequence, and can also mobilize unique DNA sequences located immediately downstream of the source element, in a process called $3^{\prime}$ transduction ${ }^{7-9}$. L1 retrotransposons can also promote the somatic trans-mobilization of Alu elements, SINE-VNTR-Alu (SVA) elements and processed pseudogenes, which are copies of mRNAs that have been reverse transcribed into DNA and inserted into the genome with the machinery of active L1 elements ${ }^{10-12}$.

Approximately $50 \%$ of human tumors contain somatic retrotranspositions of L1 elements ${ }^{7,13-15}$. Previous analyses indicate that although a fraction of somatically acquired L1 insertions in cancer may influence gene function, the majority of retrotransposon integrations in a single tumor represent passenger mutations with little or no effect on cancer development ${ }^{7,13}$. Nonetheless, L1 elements are capable of promoting other types of genomic structural alterations in the germline and somatically, in addition to canonical L1 insertion events ${ }^{16-18}$; the effect of these alterations remains largely unexplored in the context of human cancer ${ }^{19,20}$.

To further understand the roles of retrotransposons in cancer, we developed strategies to analyze the patterns and mechanisms of 
somatic retrotransposition in 2,954 cancer genomes from 38 histological cancer subtypes within the framework of the PCAWG project $^{21}$, many of which had not been evaluated for retrotransposition. On the basis of the robustness of the retrotransposition calls, we retained 296 tumors that were preliminarily excluded by the PCAWG Consortium $^{21}$ (see Methods). Our analyses identify patterns and mutational mechanisms of structural variation in human cancers that are mediated by L1 retrotransposition. We found that the aberrant integration of $\mathrm{L} 1$ retrotransposons has a relevant role in remodeling the architecture of the cancer genome in some human tumors, mainly by promoting megabase-scale deletions that, occasionally, generate genomic consequences that may promote cancer development through the removal of tumor-suppressor genes, such as $C D K N 2 A$, or trigger the amplification of oncogenes, such as CCND1.

\section{Results}

The landscape of somatic retrotransposition in a large cancer whole-genome dataset. We ran our bioinformatic pipelines (Methods and Supplementary Note) to explore somatic retrotransposition on whole-genome sequencing data from 2,954 tumors and their matched normal pairs, across 38 cancer types (Supplementary Fig. 1 and Supplementary Table 1). The analysis retrieved a total of 19,166 somatically acquired retrotranspositions that were classified into six categories (Fig. 1a and Supplementary Table 2). Comprising $98 \%(18,739$ out of 19,166$)$ of the events, L1 integrations $(14,967$ solo-L1, 3,669 L1-transductions, and 103 L1-mediated rearrangements, which mainly comprised deletions) overwhelmingly dominate the landscape of somatic retrotransposition in the PCAWG dataset (Fig. 1a,b). By contrast, elements of the lineages Alu (Supplementary Fig. 2) and SVA (comprising 130 and 23 somatic copies, respectively) and processed pseudogenes, with 274 events, represent minor categories.

The core pipeline, TraFiC-mem (Supplementary Fig. 3) -which was used to explore somatic retrotransposition in PCAWG-was validated by single-molecule whole-genome sequencing data analysis of one cancer cell line with high retrotransposition rate and its matched normal sample, confirming the somatic acquisition of 295 out of 308 retrotransposition events (false discovery rate $<5 \%$, Supplementary Fig. 4a,b). To further evaluate TraFiC-mem, we reanalyzed a mock cancer genome into which we had previously ${ }^{7}$ seeded somatic retrotransposition events at different levels of tumor clonality, and then simulated sequencing reads to the average level of coverage of the PCAWG dataset. The results confirmed a high precision ( $>99 \%$ ) of TraFiC-mem, and a recall ranging from 90 to $94 \%$ for tumor clonalities from 25 to $100 \%$, respectively (Supplementary Fig. 4c-e).

We observed marked variation in the retrotransposition rate across PCAWG tumor types (Fig. 1c and Supplementary Table 3). Overall, 35\% (1,046 out of 2,954) of all cancer genomes have at least one retrotransposition event. However, esophageal adenocarcinoma, head-and-neck squamous carcinoma, lung squamous carcinoma and colorectal adenocarcinoma are significantly enriched in somatic retrotranspositions (Mann-Whitney $U$-test, $P<0.05$; Fig. 1c,d and Supplementary Fig. 5). These four tumor types alone account for $70 \%(13,373$ out of 19,166$)$ of all somatic events in the PCAWG dataset, although they represent just 9\% (266 out of $2,954)$ of the samples. This is particularly noticeable in esophageal adenocarcinoma, in which $27 \%$ (27 out of 99 ) of the samples show more than 100 separate somatic retrotranspositions (Fig. 1c), making L1 insertions the most frequent type of structural variation in esophageal adenocarcinoma (Fig. 1e). Furthermore, retrotranspositions are the second-most frequent type of structural variants in head-and-neck squamous and colorectal adenocarcinomas (Fig. 1e). To gain insights into the genetic causes that make some cancers more prone to retrotransposition than others, we looked for associations between retrotransposition and driver mutations in cancer-related genes. This analysis revealed an increased L1 retrotransposition rate in tumors with TP53 mutations (Mann-Whitney $U$-test, $P<0.05$; Supplementary Fig. 6), and supports previous analyses that have suggested that TP53 functions to restrain mobile elements ${ }^{22,23}$. We also observe a widespread correlation between L1 retrotransposition and other types of structural variation (Spearman's $\rho=0.44$, $P<0.01$; Supplementary Fig. 7), a finding that is most likely a consequence of a confounding effect of TP53-mutated genotypes (Supplementary Fig. 6).

We identified $43 \%(7,979$ out of 18,636$)$ somatic retrotranspositions of L1 inserted within gene regions including promoters, of which 66 events hit cancer-associated genes. The analysis of expression levels in samples with available transcriptome data, revealed four genes-including the $A B L$ oncogene-with L1 retrotranspositions in the proximity of promoter regions that showed significant overexpression compared with the expression in the remaining samples of the same tumor type (Student's $t$-test, $q<0.10$; Supplementary Fig. $8 \mathrm{a}-\mathrm{c}$ ). The structural analysis of RNA-sequencing data identified instances in which portions of a somatic retrotransposition within a gene exonize, a process that sometimes involves cancer-associated genes (Supplementary Fig. 8d). In addition, we found evidence of aberrant fusion transcripts arising from the inclusion of processed pseudogenes in the target host gene and expression of processed pseudogenes landing in intergenic regions (Supplementary Fig. 8e).

Dissecting the genomic features that influence the landscape of L1 retrotranspositions in cancer. The genome-wide analysis of the distribution of somatic L1 insertions across the cancer genome revealed considerable variation in the rate of $\mathrm{L} 1$ retrotransposition (Fig. 2a and Supplementary Table 4). To understand the reasons behind such variation, we studied the association of L1 event rates with various genomic features. We first investigated whether the distribution of somatic L1s across the cancer genome could be determined by the occurrence of L1-endonuclease target-site motifs. We used a statistical approach based on negative binomial regression to deconvolute the influence of multiple overlapping genomic variables $^{24}$; this analysis showed that close matches to the motif have a 244-fold increased L1 rate, compared with non-matched motifs (Fig. 2b and Supplementary Fig. 9a). Adjusting for this effect, we found a strong association with DNA replication time; the latest-replicating quarter of the genome was 8.9-fold enriched in L1 events (95\% confidence interval, 8.25-9.71) compared with the earliest-replicating quarter (Fig. 2b,c and Supplementary Fig. 9b). Recent work $^{25}$ has shown that L1 retrotransposition has a strong cell-cycle bias, and preferentially occurs during $S$ phase. Our results are in agreement with these findings and suggest that L1 retrotransposition peaks in the later stages of nuclear DNA synthesis.

Next, we examined L1 rates in open chromatin measured using DNase hypersensitivity and, conversely, in closed heterochromatic regions by analyzing K9-trimethylated histone $\mathrm{H} 3$ (H3K9me3) ${ }^{26}$. When adjusting for the confounding effects of L1 motif content and replication time ${ }^{24}$, we found that somatic L1 events are enriched in open chromatin (1.27-fold in the top DNase hypersensitivity bin; 95\% confidence interval, 1.14-1.41; Fig. 2b) and depleted in heterochromatin (1.72-fold, 95\% confidence interval, 1.57-1.99; Fig. 2b). This finding differs from previous analyses, which have suggested that $\mathrm{L} 1$ insertions favored heterochomatin ${ }^{7}$-a discrepancy that we believe to be due to the confounding effect between heterochromatin and late-replicating DNA regions, which was not addressed in previous analyses. We also found a negative association of L1 rate with features of active transcription of chromatin, characterized by fewer L1 events at active promoters (1.63-fold; Supplementary Fig. 9c), a slight but significant reduction in L1 rates in highly expressed genes (1.25-fold lower; 95\% confidence interval, 1.16-1.34; Fig. 2b) and a further depletion at $\mathrm{H} 3 \mathrm{~K} 36 \mathrm{me} 3$ (1.90-fold reduction in the highest 


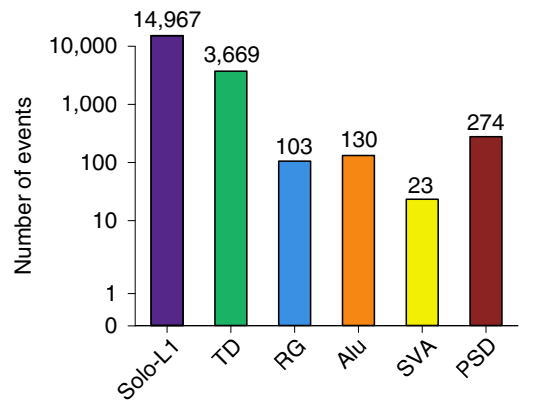

c

$$
\begin{aligned}
& \text { Number of MEI } \\
& \text { Enrichment ( } z \text {-score) }
\end{aligned}
$$

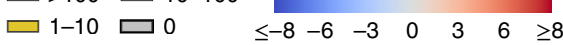

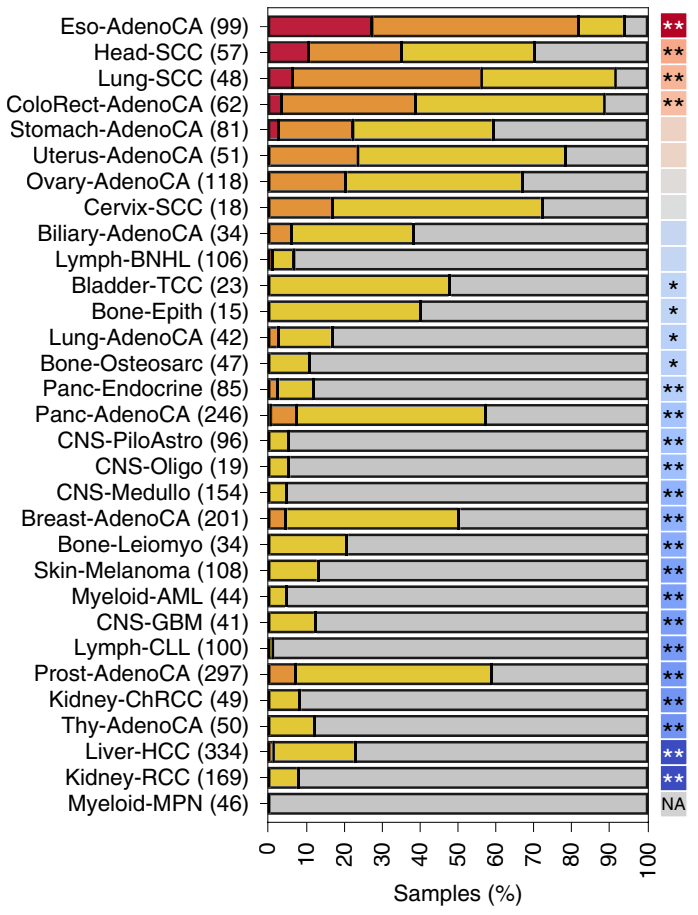

b
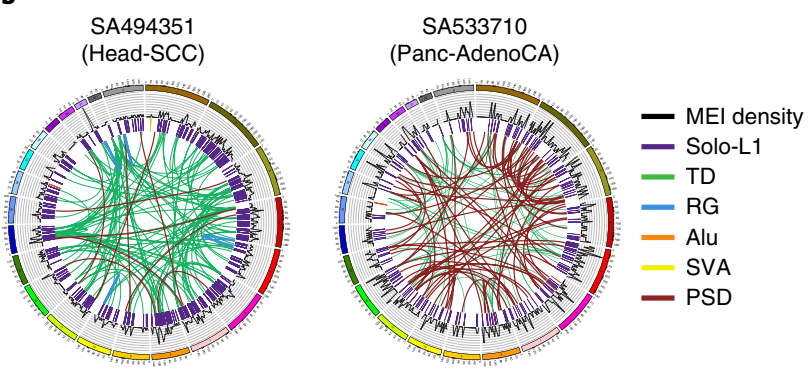

d

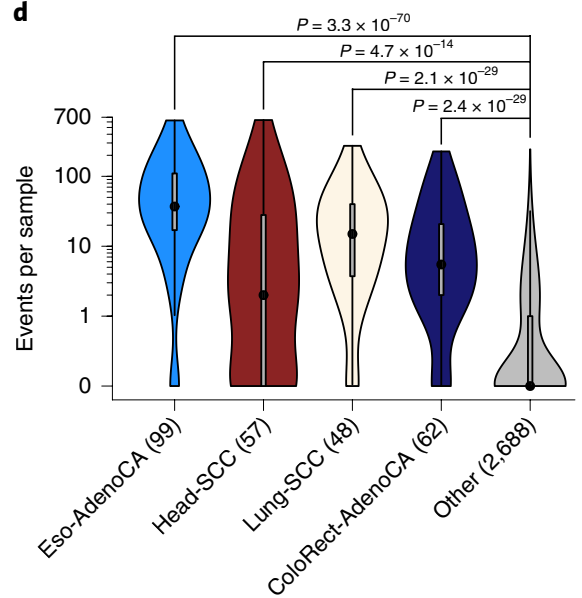

e

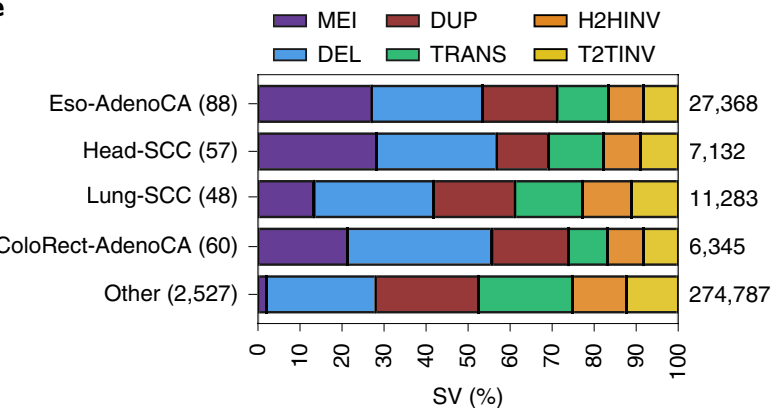

Fig. 1 | Landscape of somatic retrotransposition across human cancers. a) Number of somatic retrotransposition events identified in 2,954 cancer genomes across six categories: solo-L1, L1-mediated transductions (TD), L1-mediated rearrangements (RG), Alu, SVA and pseudogenes (PSD). b, Left, circos plot showing a head-and-neck tumor (Head-SCC) with high retrotransposition rate (638 somatic events). Right, a single pancreatic adenocarcinoma sample harboring around 26\% (70 out of 274) of all processed pseudogenes identified in the PCAWG cohort. Chromosome ideograms are shown around the outer ring with individual rearrangements represented as arcs; colors match the type of rearrangement. c, For 31 PCAWG cancer types with sample size of $n \geq 15$, data show the proportion of tumor samples with $>100$ (red), 10-100 (orange), 1-10 (yellow) and 0 (gray) somatic retrotranspositions. The number of samples analyzed for each tumor type is shown in parentheses. Retrotransposition enrichment or depletion for each tumor type together with the level of significance (zero-inflated negative binomial regression) is shown. ${ }^{\star} P<0.05,{ }^{\star} P<0.01$. NA, not applicable. d, Distribution of retrotransposition events per sample across the four tumor types significantly enriched in somatic retrotranspositions; the remaining tumors are grouped into 'Other'. The number of samples from each group is shown in parentheses; point, median; box, 25th to 75th percentiles (interquartile range); whiskers, data within $1.5 \times$ the interquartile range. $P$ values indicate significance from a two-tailed Mann-Whitney $U$-test. The $y$ axis is shown on a logarithmic scale. e, For the same four tumor types in $\mathbf{d}$, the fraction of structural variants (SV) belonging to six classes is shown: mobile element insertions (MEI), deletions (DEL), duplications (DUP), translocations (TRANS), head-to-head inversions (H2HINV) and tail-to-tail inversions (T2TINV). The total number of structural variants per cancer type is indicated on the right side of the panel.

tertile; 95\% confidence interval, 1.59-2.29; Fig. 2b), a mark of actively transcribed regions deposited in the body and at the $3^{\prime}$ end of active genes ${ }^{26}$. Further details on these associations are shown in Supplementary Fig. 9c-e and described in the Supplementary Note.

The contribution of $\mathrm{L} 1$ source elements to the pan-cancer retrotransposition burden. We used somatically mobilized L1 3' transduction events to trace L1 activity to specific source elements? This strategy revealed 124 germline L1 loci in the human genome that are responsible for most of the genomic variation generated by retrotransposition in the PCAWG dataset ${ }^{7,21}$ (Supplementary Table 5). To our knowledge, 52 of these loci represent previously unreported source elements in human cancer ${ }^{21}$. We analyzed the relative contribution of individual source elements to retrotransposition burden across cancer types, and found that retrotransposition is generally dominated by five hot-L1 source elements that alone give rise to half of all somatic transductions (Fig. 3a). This analysis revealed a dichotomous pattern of hot-L1 activity, with source 

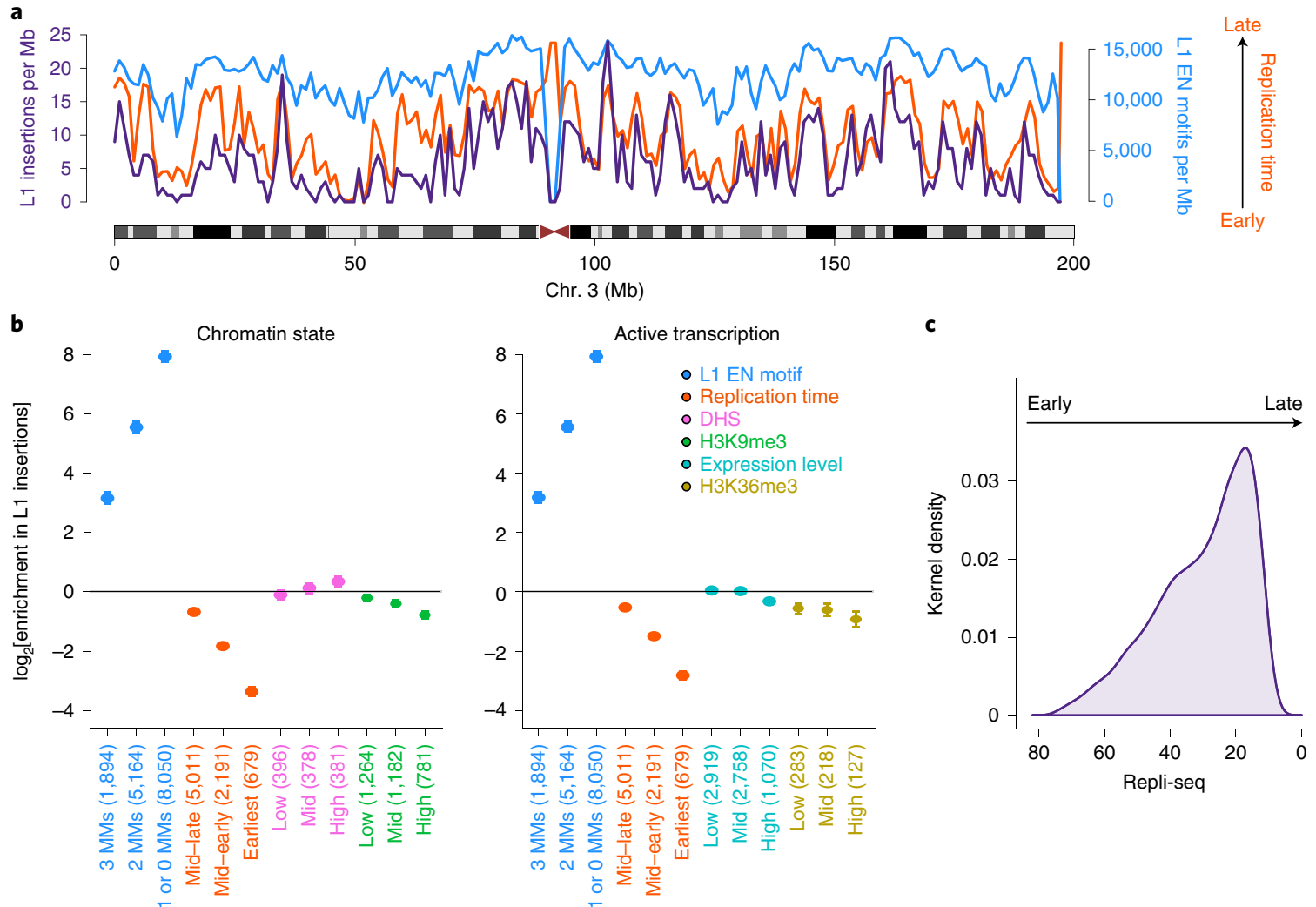

Fig. 2 | Distribution of L1 somatic insertions across the cancer genome and its association with genome organization features. Genome-wide analysis of the distribution of 15,906 somatic L1 insertions, which include solo-L1 and L1 transductions with a 3'-poly(A) breakpoint characterized to base-pair resolution. $\mathbf{a}$, The L1 insertion rate (purple) is shown together with the L1 endonuclease (EN) motif density (blue) and replication timing (orange). The data are represented per 1-Mb window. For illustrative purposes, only chromosome 3 is shown. $\mathbf{b}$, Association between L1 insertion rate and multiple predictor variables at single-nucleotide resolution. Enrichment scores (thick dots) are adjusted for multiple covariates and compare the L1 insertion rate in bins 1-3 for a particular genomic feature (L1 endonuclease motif, replication timing, open chromatin, histone marks and expression level) versus bin 0 of the same feature, which therefore always has log-transformed enrichment $=0$ by definition and is not shown. The error bars represent $95 \%$ confidence intervals. The number of observations per bin is provided in parentheses. MMs, the number of mismatches with respect to the consensus L1 endonuclease motif (see Supplementary Note). Heterochromatic regions and transcription elongation are defined based on $\mathrm{H} 3 \mathrm{~K} 9 \mathrm{me} 3$ and $\mathrm{H} 3 \mathrm{~K} 36 \mathrm{me} 3$ histone marks. Accessible chromatin is measured through DNase hypersensitivity. c, L1 insertion density, using kernel density estimate (KDE), along the replication timing spectrum. DNA replication timing is expressed on a scale from 80 (early) to 0 (late).

elements that we have termed Strombolian and Plinian, given their similarity to these two types of volcanoes (Fig. 3b). Strombolian source elements are relatively indolent and produce small numbers of retrotranspositions in individual tumor samples, although they are often active and contribute substantially to overall retrotransposition in the PCAWG dataset. By contrast, Plinian elements are rarely active across tumors, but in these isolated cases, their activity is fulminant, causing large numbers of retrotranspositions.

At the individual tumor level, although we observed that the number of active source elements in a single cancer genome varied from 1 to 22 , typically only 1 to 3 loci were operative (Fig. 3c). There is a correlation between somatic retrotranspositions and the number of active germline L1 source elements among PCAWG samples (Fig. 3d); this is likely one of the factors that explains why esophageal adenocarcinoma, lung and head-and-neck squamous carcinoma account for higher retrotransposition rates-in these three tumor types we also observed higher numbers of active germline L1 loci (Fig. 3c). Occasionally, somatic L1 integrations that retain their full length may also act as a source for subsequent somatic retrotransposition events ${ }^{7,27}$, and may reach high activity rates, leading them to dominate retrotransposition in a given tumor. For example, in a remarkable head-and-neck tumor sample, SA197656, we identified one somatic L1 integration at 4 p16.1 that then triggered 18 transductions from its new site, with the next most active element being a germline L1 locus at 22q12.1, which accounted for 15 transductions (Supplementary Table 5).

Genomic deletions mediated by somatic $\mathrm{L} 1$ retrotransposition. In cancer genomes with high somatic L1 activity rates, we observed that some L1 retrotransposition events followed a distinctive pattern that consisted of a single cluster of reads, associated with copy-number loss, for which the mates unequivocally identified one extreme of a somatic L1 integration with, apparently, no local, reciprocal cluster that supported the other extreme of the L1 insertion (Fig. 4a). Analysis of the associated copy-number changes identified the missing L1 reciprocal cluster at the far end of the copy-number loss, indicating that this pattern represents a deletion that occurred in conjunction with the integration of an L1 retrotransposon (Fig. 4b; see the Supplementary Note for additional information on how to interpret the paired-end mapping data from this and other figures). These rearrangements-called L1-mediated deletionshave been observed to occur somatically with engineered L1s in cultured human cells ${ }^{16,17}$ and naturally in the brain ${ }^{18}$, and are most likely the consequence of an aberrant mechanism of L1 integration.

We developed specific algorithms to systematically identify L1-mediated deletions, and applied these methods across all PCAWG tumors. We identified 90 somatic events that matched the patterns described above, causing deletions of different size, which ranged 
a

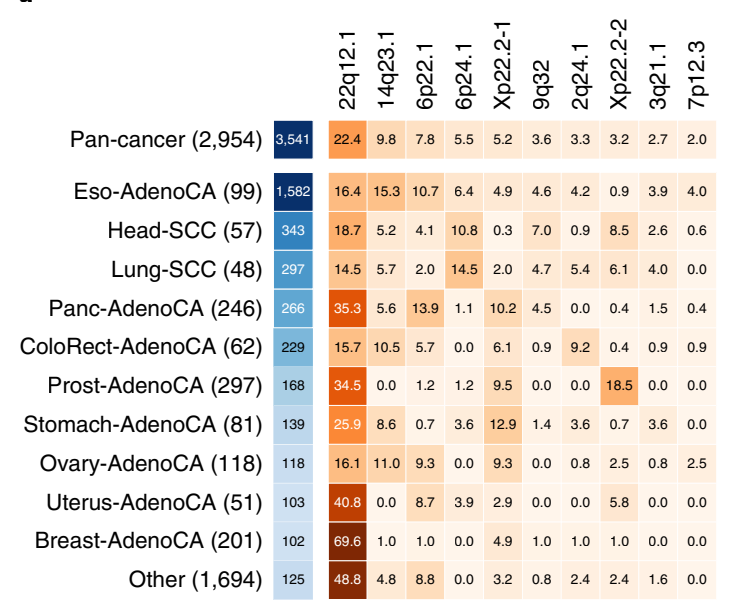

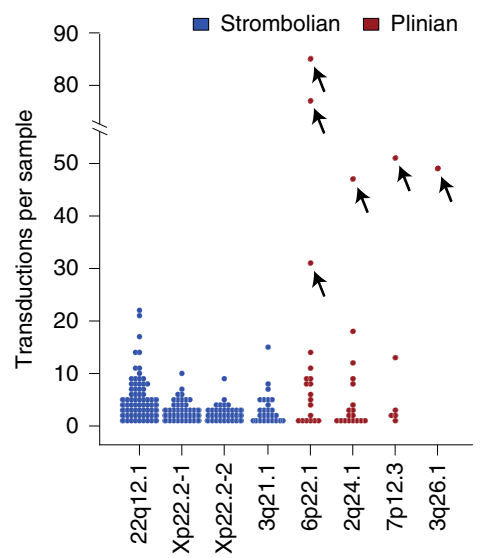
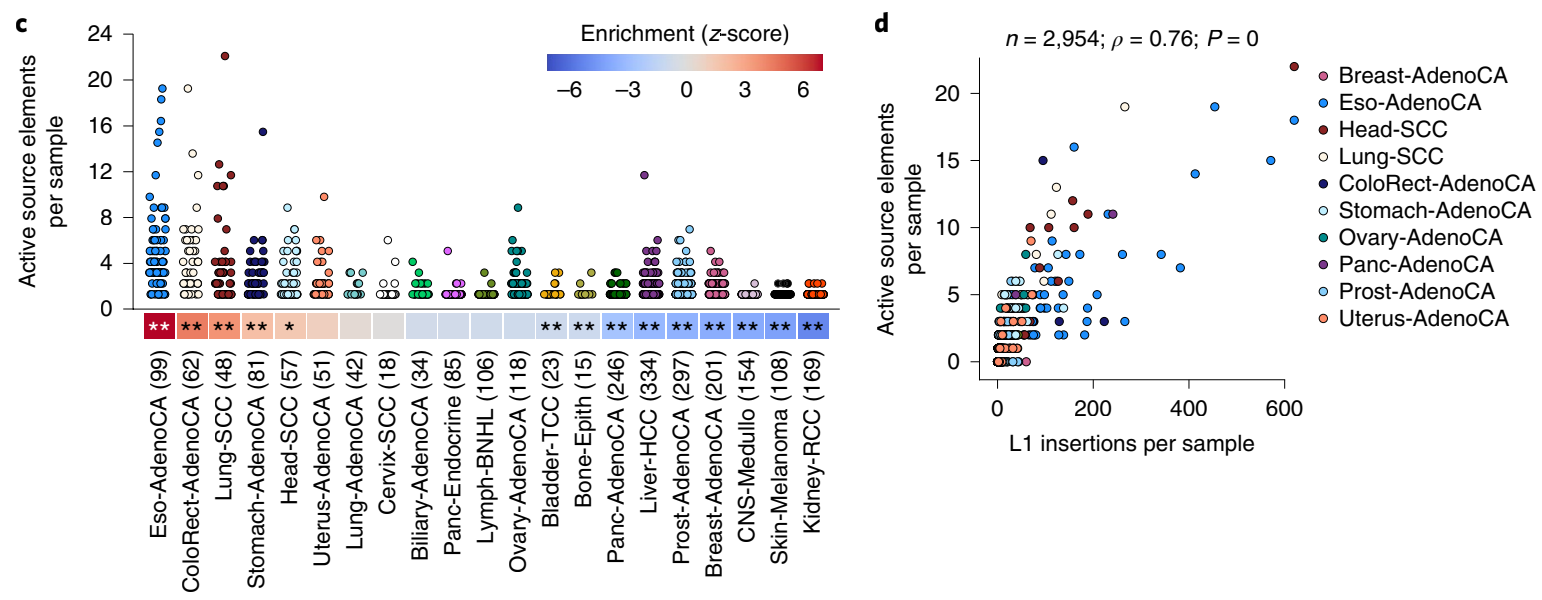

Fig. 3 | The dynamics of $\mathbf{L} 1$ source-element activity in human cancer. $\mathbf{a}$, The total number of transductions identified for each cancer type is shown as a blue-colored scale. The sample size for each tumor type is shown in parentheses. Contribution of each source element is defined as the proportion of the total number of transductions from each cancer type that is explained by each source locus. Only the top ten contributing source elements are shown, while the remaining are grouped into the category 'Other'. b, Two extreme patterns of hot-L1 activity, Strombolian (blue) and Plinian (red), were identified. Dots show the number of transductions promoted by each source element in a given tumor sample. Arrows highlight violent eruptions (that is, strong peaks of somatic activity) in particular samples. c, Number of active germline L1 source elements per sample, across cancer types with source element activity. A source element is considered to be active in a given sample if it promotes at least one transduction. The enrichment or depletion of the number of active source elements for each tumor type together with the level of significance (zero-inflated negative binomial regression) is shown. ${ }^{\star} P<0.05$, ${ }^{\star \star} P<0.01$. The number of samples analyzed for each tumor type is shown in parentheses. $\mathbf{d}$, Correlation between the number of somatic L1 insertions and the number of active germline L1 source elements in PCAWG samples. Each dot represents a tumor sample and colors match cancer types. Sample sizes $(n)$, together with Spearman's $\rho$ and $P$ values are shown above the panel.

in size from around $0.5 \mathrm{~kb}$ to $53.4 \mathrm{Mb}$ (Fig. $4 \mathrm{c}$ and Supplementary Table 6). The reconstruction of the sequence at the breakpoint junctions in each case supports the presence of an L1-elementor L1-transduction-sequence and its companion polyadenylate tract, indicative of passage through an RNA intermediate. No target site duplication was found, which is also the typical pattern for L1-mediated deletions ${ }^{17}$. One potential mechanism for these events is that a molecule of L1 cDNA pairs with a distant $3^{\prime}$ overhang from a pre-existing double-strand DNA break generated upstream of the initial integration site, and the DNA region between the break and the original target site is subsequently removed by aberrant repair ${ }^{17}$ (Fig. 4d). Indeed, in 75\% (47 out of 63) of L1-mediated deletions with a $5^{\prime}$-end breakpoint characterized to base-pair resolution, the analysis of the sequences at the junction revealed short (1-5 bp long, with median at $3 \mathrm{bp}$ ) microhomologies between the pre-integration site and the $5^{\prime} \mathrm{L} 1$ sequence integrated right there (Supplementary Table 6). Furthermore, we found 14\% (9 out of 63) instances in which short insertions (1-33bp long, with median at $9 \mathrm{bp}$ ) are found at the $5^{\prime}$-breakpoint junction of the insertion. Both signatures are consistent with a non-homologous end-joining mechanism ${ }^{28}$, or other type of microhomology-mediated repair, for the $5^{\prime}$-end attachment of the L1 cDNA to a $3^{\prime}$ overhang from a pre-existing double-strand DNA break located upstream. L1-mediated deletions in which microhomologies or insertions are not found may follow alternative models $s^{17,29-31}$.

To confirm that these rearrangements are mediated by the integration of a single intervening retrotransposition event, we explored the PCAWG dataset for somatic L1-mediated deletions in which the L1 sequences at both breakpoints of the deletion could unequivocally be assigned to the same L1 insertion. These include small deletions and associated $\mathrm{L} 1$ insertions that were shorter than the library size, allowing sequencing read pairs to overlay the entire structure. For example, in a lung tumor sample, SA313800, we identified a deletion involving a 1-kb region of $19 q 12$ with hallmarks of 


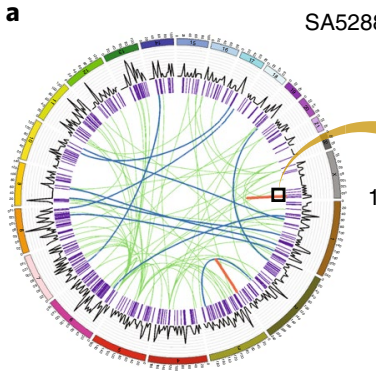

- MEI density

Solo-L1

TD

PSD

L1-mediated deletion
Single L1 cluster at Chr. X

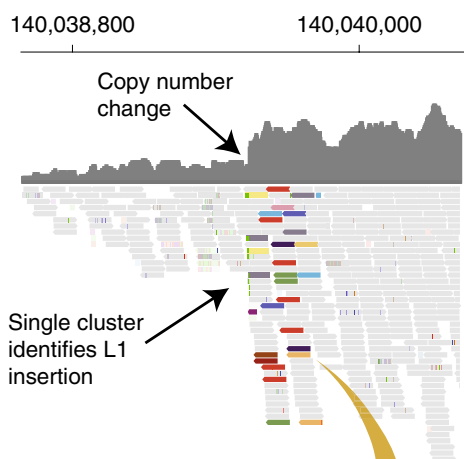

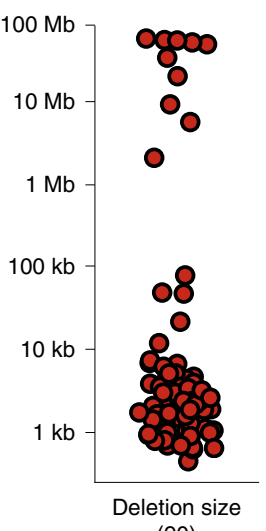

(90)
L1-mediated deletion model

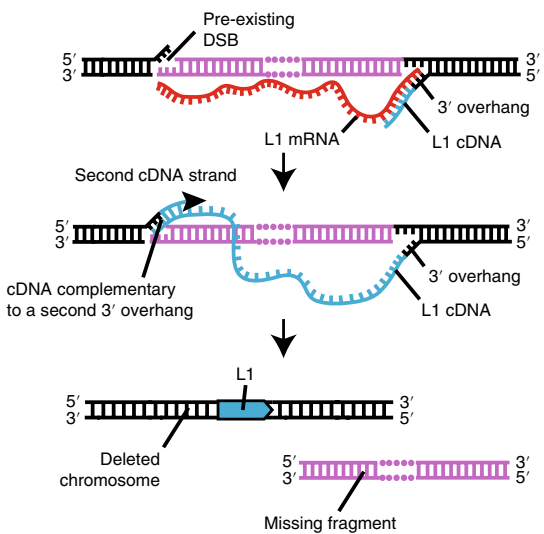

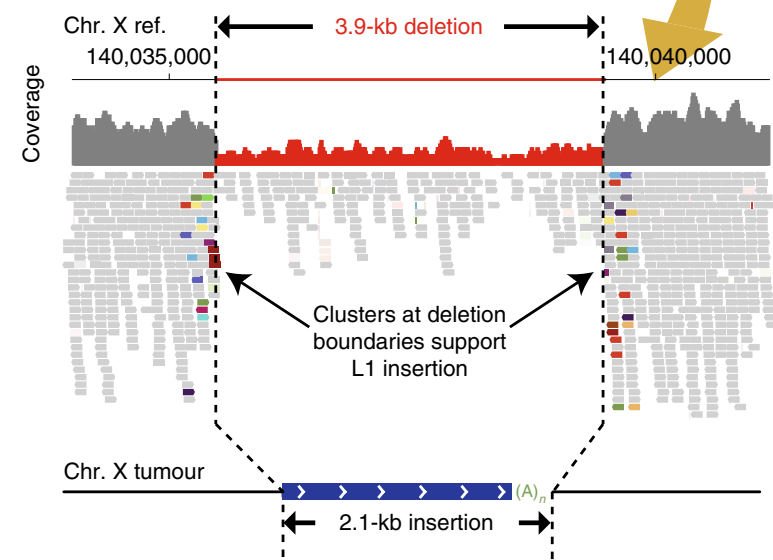

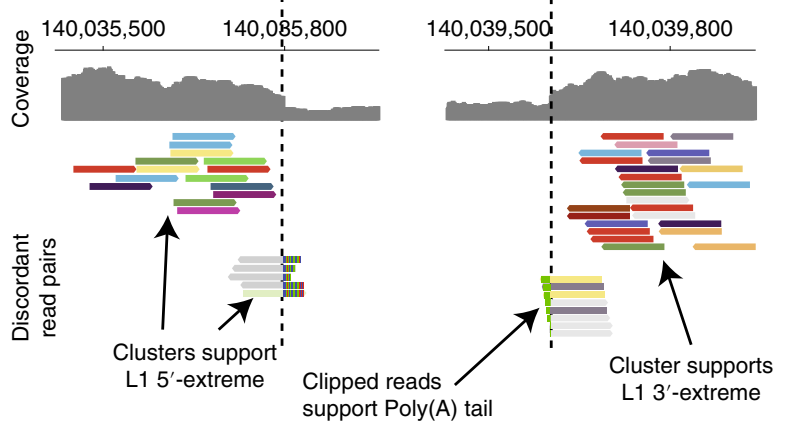

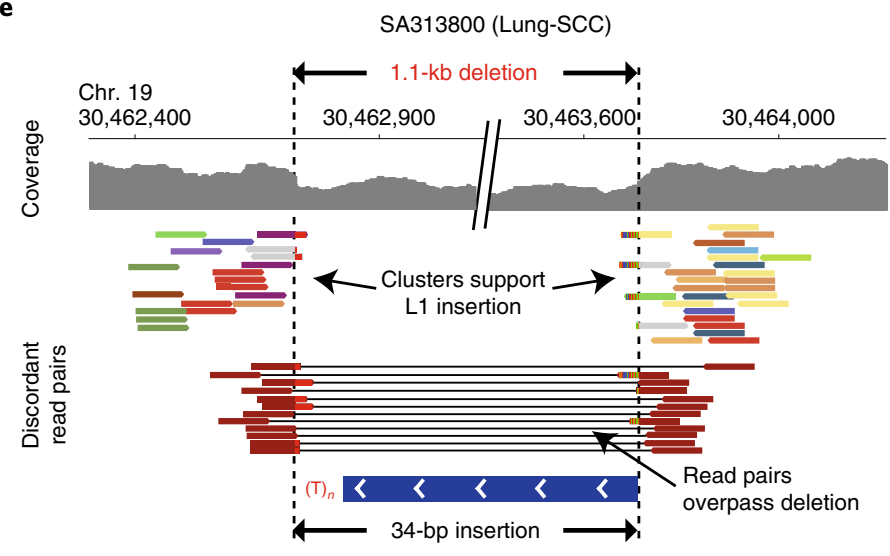

f

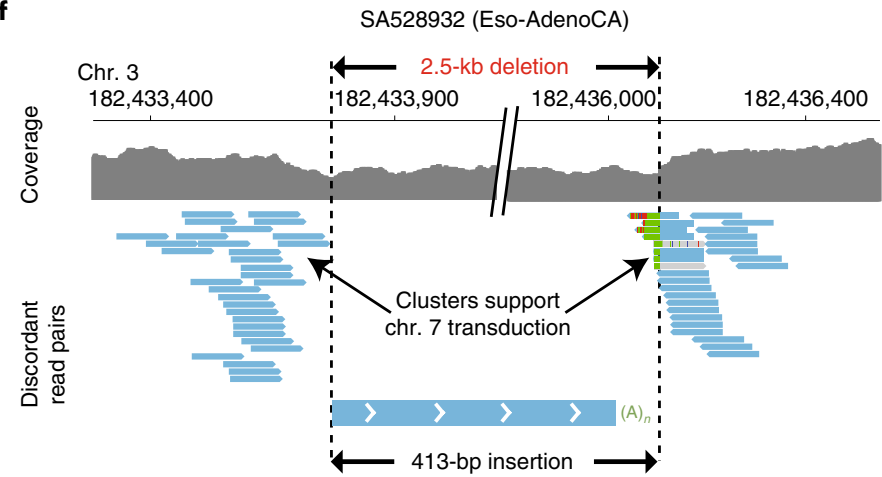

Fig. 4 | The hallmarks of somatic L1-mediated deletions revealed by copy-number and paired-end mapping analysis. a, In esophageal adenocarcinoma sample SA528802, we found a single cluster of reads on chromosome X, which is associated with one breakpoint of a copy-number loss, and for which the mates unequivocally identified one extreme of a somatic L1 integration. Paired-end reads are colored by the chromosome on which their mates can be found. Different colors for different reads from the same cluster indicate that mates are mapping a repetitive element. $\mathbf{b}$, Analysis of the associated copy-number change on chromosome $X$ identifies the missing L1 reciprocal cluster at the second breakpoint of the copy-number loss, and reveals a 3.9-kb deletion that occurs in conjunction with the integration of a 2.1-kb L1 somatic insertion. $(A)_{n}$ and $(T)_{n}$ represent poly $(A)$ and poly $(T)$ tails, respectively. c, Model of L1-mediated deletion. The integration of an L1 mRNA starts with L1-endonuclease cleavage promoting a 3' overhang for reverse transcription. The cDNA (-) strand invades a second 3' overhang from a pre-existing double-strand break upstream of the initial integration site. d, Distribution of the sizes of 90 L1-mediated deletions identified in the PCAWG dataset. e, In lung squamous carcinoma sample SA313800, a 34-bp truncated L1 insertion promotes a 1.1-kb deletion on chromosome 19. Because the L1 insertion was so short, we also identified discordant read pairs that span the L1 event and support the deletion. f, In esophageal adenocarcinoma sample SA528932, the integration on chromosome 3 of a 413 -bp orphan L1 transduction from chromosome 7 causes a 2.5-kb deletion, which is supported by two clusters of discordant read pairs for which the mates map onto the transduced region of chromosome 7.

being generated by an L1 element (Fig. 4e). In this rearrangement, we found two different types of discordant read pairs at the deletion breakpoints: one cluster that supported the insertion of an L1 element and a second that spanned the L1 event and supported the deletion. Another type of L1-mediated deletion that could unequivocally be assigned to a single L1 insertion event is represented 

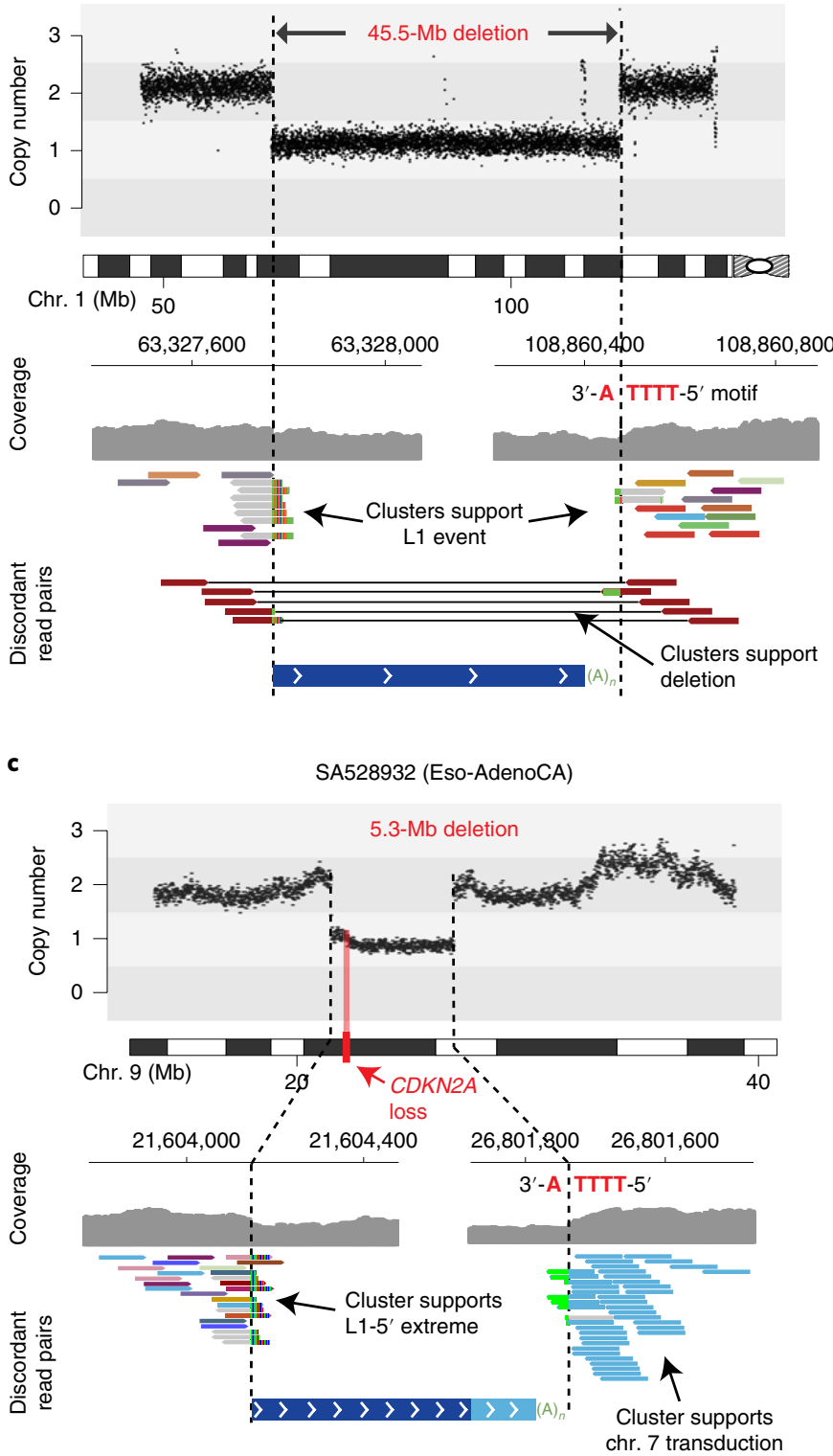
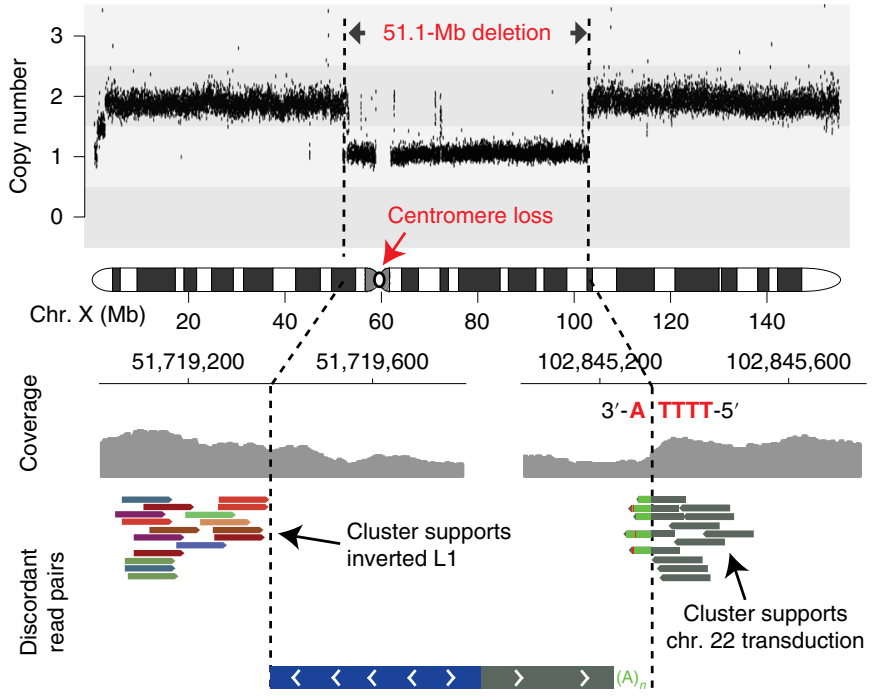

d

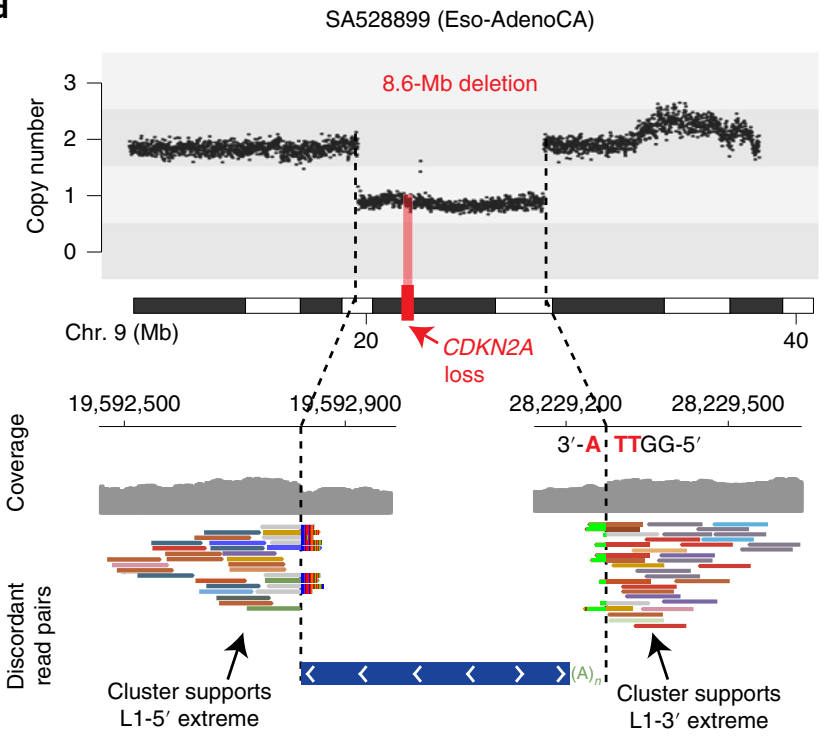

Fig. 5 | Somatic integration of L1 causes loss of megabase-size interstitial chromosomal regions in cancer. a, In esophageal adenocarcinoma sample SA528901, a 45.5-Mb interstitial deletion on chromosome 1 is generated after integration of a short L1 event. We observed a pair of clusters of discordant read pairs for which the mates support both extremes of the L1 insertion. Because the L1 element event is smaller than the library insert size, we also identified read pairs that span the L1 event and support the deletion. The L1-endonuclease 5'-TTTT/A-3' motif identifies a target-primed reverse transcription (TPRT) L1-integration mechanism. b, In esophageal tumor sample SA313800, a partnered transduction ${ }^{7}$ (that is, the transduced region and its companion L1 source element) from chromosome 22 is integrated on chromosome $\mathrm{X}$, promoting a 51.1-Mb deletion that removes the centromere. One negative cluster (green reads) supports a small region transduced from chromosome 22. c, L1-mediated deletions promote the loss of tumor-suppressor genes. In esophageal tumor sample SA528932, the somatic integration on chromosome 9 of a partnered transduction from chromosome 7, promotes a 5.3-Mb deletion that involves the loss of one copy of the tumor-suppressor gene CDKN2A. We observed a positive cluster of reads for which the mates map onto the $5^{\prime}$ extreme of an $\mathrm{L} 1$, and a negative cluster that contains split reads that match a poly $(\mathrm{A})$ region and for which the mates map onto a region that is transduced from chromosome 7 (light blue). d, In a second esophageal adenocarcinoma sample, SA528899, the integration of an L1 retrotransposon generates an $8.6-\mathrm{Mb}$ deletion that involves the same tumor-suppressor gene, CDKN2A. The sequencing data reveal two clusters-positive and negative-for which the mates support the L1 event.

by those deletions generated by the integration of orphan L1 transductions. These transductions represent fragments of unique DNA sequence located downstream of an active L1 locus, which are mobilized without the companion L1 (refs. ${ }^{7,15}$ ). For example, in one esophageal tumor sample, SA528932, we found a deletion of $2.5 \mathrm{~kb}$ on chromosome 3 mediated by the orphan transduction of a sequence downstream of an L1 locus on chromosome 7 (Fig. 4f).
Owing to the unavailability of PCAWG DNA specimens, we performed a validation of 16 additional somatic L1-mediated deletions that were identified by TraFiC-mem in two head-and-neck cancer cell lines with high retrotransposition rates, NCI-H2009 and $\mathrm{NCI}-\mathrm{H} 2087^{7}$. We carried out two independent validation approaches, including PCR followed by single-molecule sequencing of amplicons, and Illumina whole-genome sequencing 

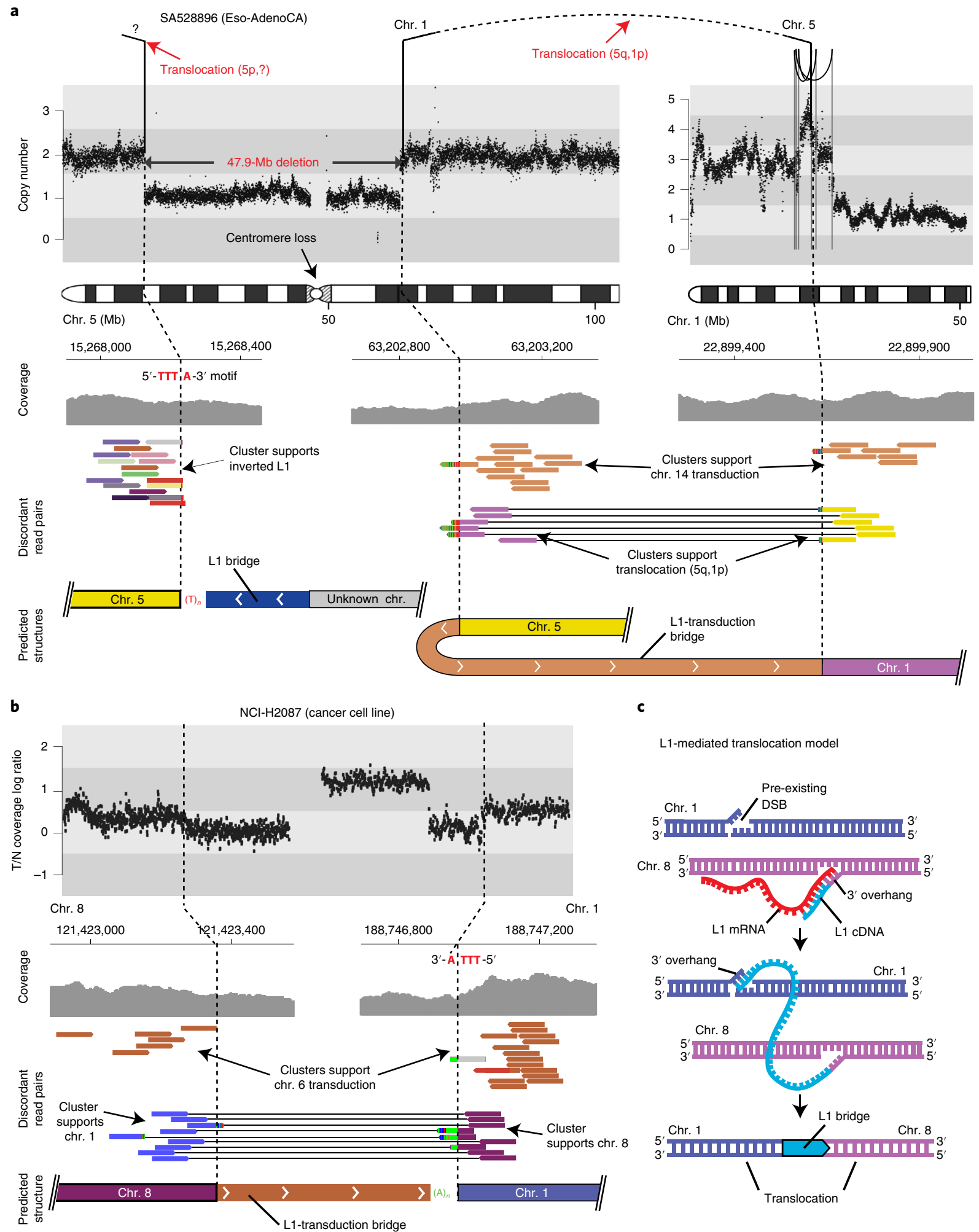

L1-mediated translocation model

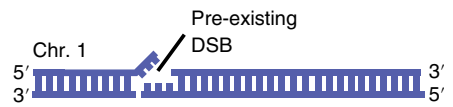

Chr. $8{ }_{3}^{5}$, 'IIIIIIIIIIIIIIII

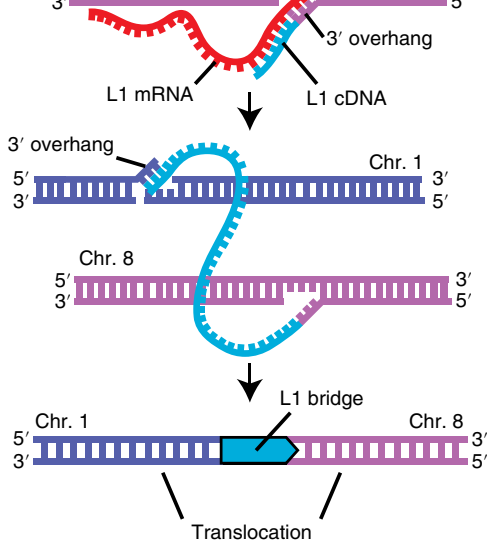

Fig. 6 | Somatic L1 integration promotes translocations in human cancers. a, In esophageal adenocarcinoma sample SA528896, two separate L1 events mediate interchromosomal rearrangements. In the first, an L1 transduction from a source element on chromosome 14q23.1 bridged an unbalanced translocation from chromosome $1 p$ to $5 q$. A second somatic retrotransposition event bridged from chromosome $5 p$ to an unknown part of the genome, completing a 47.9-Mb interstitial copy-number loss on chromosome 5 that removes the centromere. $\mathbf{b}$, In a cancer cell line, $\mathrm{NCl}-\mathrm{H} 2087$, we found an interchromosomal translocation, between chromosomes 8 and 1, mediated by a region transduced from chromosome 6 , which acts as a bridge and joins both chromosomes. We observed two read clusters, positive and negative, that demarcate the boundaries of the rearrangement, for which the mates support the transduction event. In addition, two reciprocal clusters span the insertion breakpoints, supporting the translocation between chromosomes 8 and 1. c, A model for megabase-size L1-mediated interchromosomal rearrangements. L1-endonuclease cleavage promotes a 3' overhang in the negative strand, retrotranscription starts and the cDNA (-) strand invades a second $3^{\prime}$ overhang from a pre-existing double-strand break on a different chromosome, leading to translocation. 
using mate-pair libraries with long insert size ( $3 \mathrm{~kb}$ and $10 \mathrm{~kb})$. The results confirmed the somatic status of the rearrangements and a single L1-derived retrotransposition as the cause of the associated copy-number loss (Supplementary Figs. 10-12 and Supplementary Table 7).

Analysis of L1 3'-extreme insertion breakpoint sequences from L1-mediated deletions found in the PCAWG dataset revealed that $82 \%$ (74 out of 90 ) of the L1 events that caused deletions preferentially inserted into sequences that resemble L1-endonuclease consensus cleavage sites (for example, $5^{\prime}$-TTTT/A-3' and related sequences $^{32}$ ) (Supplementary Table 6). This confirms that the L1 machinery, through a target-primed reverse-transcription mechanism, is responsible for the integration of most of the $\mathrm{L} 1$ events that cause neighboring DNA loss ${ }^{32}$. Notably, in 16\% (14 out of 90) of the events endonucleotidic cleavage occurred at the phosphodiester bond between a T and $\mathrm{G}$ instead of between the standard $\mathrm{T}$ and A site. In addition, we observed 8\% (7 out of 90) instances in which the endonuclease motif was not found and the integrated element was truncated at both the $5^{\prime}$ and $3^{\prime}$ ends, suggesting that a small fraction of L1-associated deletions are the consequence of an L1-endonuclease-independent insertion mechanism ${ }^{30-32}$. Whatever mechanism of L1 integration is effective in each case, taken together, these data indicate that the somatic integration of L1 elements induces the associated deletions.

Megabase-size L1-mediated deletions cause loss of tumorsuppressor genes. Most L1-mediated deletions ranged from a few hundred to thousands of base pairs, although occasionally megabase-long regions of a chromosome were deleted (Fig. $4 \mathrm{c}$ and Supplementary Table 6). For example, in esophageal tumor sample SA528901, we found a $45.5-\mathrm{Mb}$ interstitial deletion that involved the p31.3-p13.3 regions of chromosome 1 (Fig. 5a), in which both breakpoints of the rearrangement showed the hallmarks of a deletion mediated by integration of an L1 element. Here, the L1 element is $5^{\prime}$ truncated, which generated a small $\mathrm{L} 1$ insertion, allowing a fraction of the sequencing read pairs to span both breakpoints of the rearrangement. This unequivocally supports the model that the observed copy-number change is indeed a deletion mediated by retrotransposition of an L1 element. Similarly, in a lung tumor sample, SA313800, we found an interstitial L1-mediated deletion that induced the loss of $51.1 \mathrm{Mb}$ from chromosome $\mathrm{X}$, which included the centromere (Fig. 5b).

L1-mediated deletions were, on occasion, driver events and caused the loss of tumor-suppressor genes. In esophageal tumor sample SA528932, the integration of an L1 transduction from chromosome $7 \mathrm{p} 12.3$ to the short arm of chromosome 9 caused a $5.3-\mathrm{Mb}$ clonal deletion that involved the 9p21.3-9p21.2 region. This led to the loss of one copy of a key tumor-suppressor gene, CDKN2A (Fig. 5c), which is deleted in many cancer types including esophageal tumors ${ }^{33-36}$. Notably, the sequencing data revealed a somatic transduction that arose from this L1 element at its new insertion site, demonstrating that L1 events that promote deletions can be competent for retrotransposition (Supplementary Fig. 13). In a second esophageal tumor sample, SA528899, an L1 element integrated into chromosome 9 promoted an $8.6-\mathrm{Mb}$ clonal deletion that encompasses the 9p22.1-9p21.1 region that removes one copy of the same tumor-suppressor gene, CDKN2A (Fig. 5d). Thus, L1-mediated deletions have clear oncogenic potential.

L1 retrotransposition generates other types of structural variation in human tumors. Somatic retrotransposition can also be involved in mediating or repairing more complex structural variants. In one esophageal tumor sample, SA528896, two separate L1-mediated structural variants were present within a complex cluster of rearrangements (Fig. 6a). In the first, an L1 transduction from a source element on chromosome $14 \mathrm{q} 23.1$ bridged an unbalanced translocation from chromosome $1 \mathrm{p}$ to $5 \mathrm{q}$. A second somatic retrotransposition event bridged from chromosome $5 p$ to an unknown part of the genome, completing a large interstitial copy-number loss on chromosome 5 that involves the centromere. This case suggests that retrotransposon transcripts and their reverse-transcriptase machinery can mediate breakage and repair of complex dsDNA breaks, spanning two chromosomes.

To explore this further, we identified single-L1 clusters with no reciprocal cluster in the cancer cell lines that were sequenced by using mate pairs with $3 \mathrm{~kb}$ and $10 \mathrm{~kb}$ inserts. Such events may correspond to hidden genomic translocations leading to the linkage of two different chromosomes, in which L1 retrotransposition is involved. One of the samples, NCI-H2087, showed translocation breakpoints at $1 \mathrm{q} 31.1$ and $8 \mathrm{q} 24.12$, both of which had the hallmarks of L1-mediated deletions, for which the mate-pair sequencing data identified an orphan L1 transduction from chromosome 6 p24 that bridged both chromosomes (Fig. 6b). The configuration has also been confirmed by using long-read single-molecule sequencing (Supplementary Fig. 11). This interchromosomal rearrangement is likely mediated by the aberrant operation of L1-integration mechanism, in which the L1-transduced cDNA is wrongly paired with a second $3^{\prime}$ overhang from a pre-existing double-strand break generated in a second chromosome ${ }^{32}$ (Fig. 6c).

We also found evidence that L1 integrations can cause duplications of large genomic regions in human cancer. In esophageal tumor sample SA528848 (Fig. 7a), we identified two independent read clusters that support the integration of a small L1 event, coupled with a coverage drop at both breakpoints. Copy-number analysis revealed that the two L1 clusters demarcate the boundaries of a $22.6-\mathrm{Mb}$ duplication that involves the $6 \mathrm{q} 14.3-\mathrm{q} 21$ region, suggesting that the L1 insertion could be the cause of such rearrangement by bridging sister chromatids during or after DNA replication (Fig. 7b). The analysis of the rearrangement data at the breakpoints identified read pairs that traverse the length of the L1 insertion breakpoints, and the L1-endonuclease motif is the L1 $3^{\prime}$ insertion breakpoint, both confirming a single L1 event as the cause of a tandem duplication (Fig. 7a). Notably, this duplication increases the copy number of the cyclin $\mathrm{C}$ gene, $C \mathrm{CNC}$, which is dysregulated in some tumors ${ }^{37}$.

Fig. 7 | Somatic L1 integration promotes duplications of megabase-scale regions in human cancers. a, In esophageal adenocarcinoma sample SA528848, we found a 22.6- $\mathrm{Mb}$ tandem duplication on the long arm of chromosome 6 . The analysis of the sequencing data at the boundaries of the rearrangement breakpoints reveals two clusters of discordant read pairs for which the mates support the involvement of an L1 event. Because the L1 element was shorter than the library size, we also found two reciprocal clusters that aligned $22.6 \mathrm{Mb}$ apart on the genome and in opposite orientation, spanning the insertion breakpoints and confirming the tandem duplication. An L1-endonuclease 5'-TTT/A-3' degenerate motif was found. b, Large direct tandem duplications can be generated if the cDNA (-) strand invades a second 3' overhang from a pre-existing double-strand break that occurred on a sister chromatid, and downstream to the initial integration site locus. c, In lung tumor sample SA313800, a small L1 insertion causes a 79.6-Mb duplication of the 14q arm through the induction of a fold-back inversion rearrangement. The analysis of the sequencing data at the breakpoint revealed two clusters of discordant read pairs (multi-colored reads) with the same orientation, aligning close together ( $5.5 \mathrm{~kb}$ apart) and demarcating a copy-number change for which the sequencing density is much greater on the right half of the rearrangement than the left. Both clusters of multi-colored reads support the integration of an L1. d, L1-mediated fold-back inversion model. 
L1-mediated rearrangements can induce breakage-fusion-bridge cycles that trigger oncogene amplification. L1 retrotranspositions can also induce genomic instability by triggering breakage-fusionbridge cycles. This form of genetic instability starts with end-to-end fusion of broken sister chromatids, and lead to a dicentric chromosome that forms an anaphase bridge during mitosis. Classically, the end-to-end chromosome fusions are thought to arise from telomere attrition $^{38-40}$. We found, however, that somatic retrotransposition a

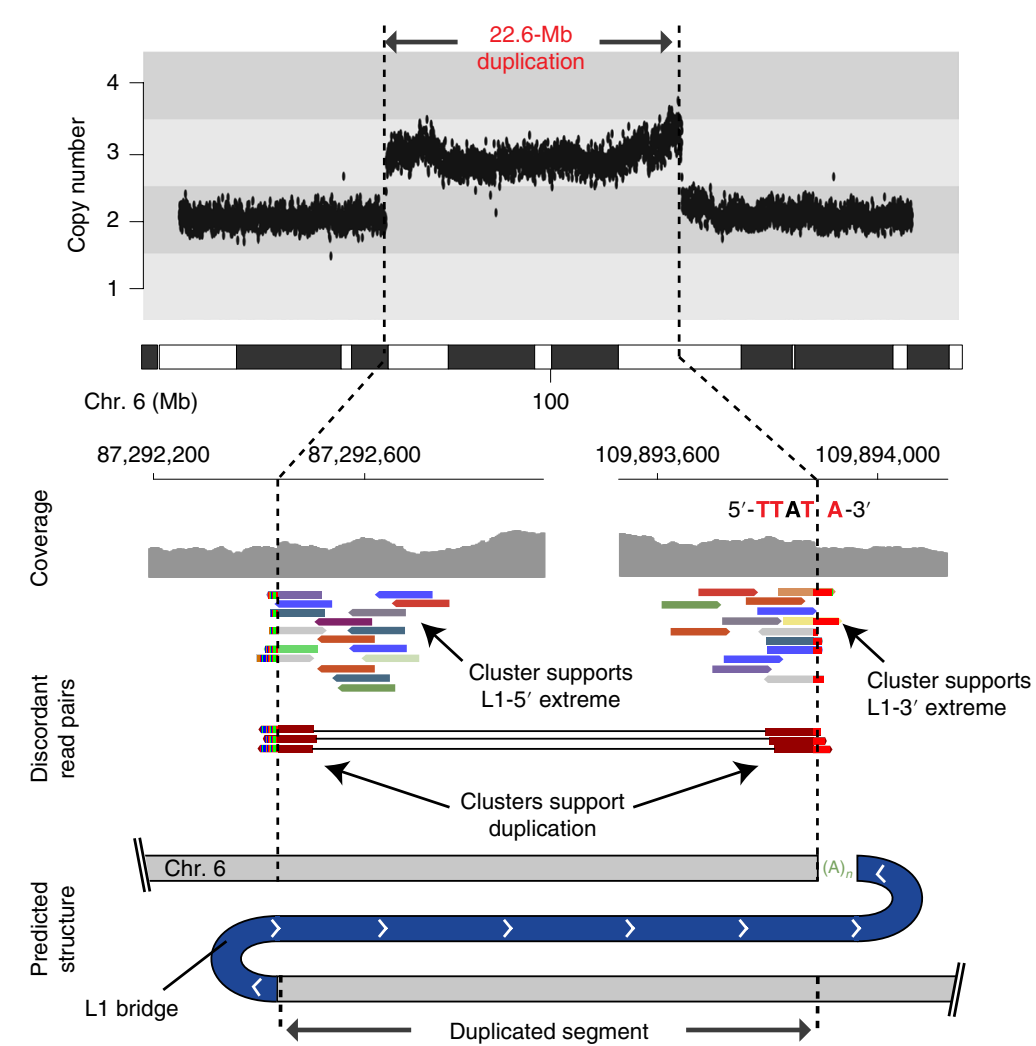

c

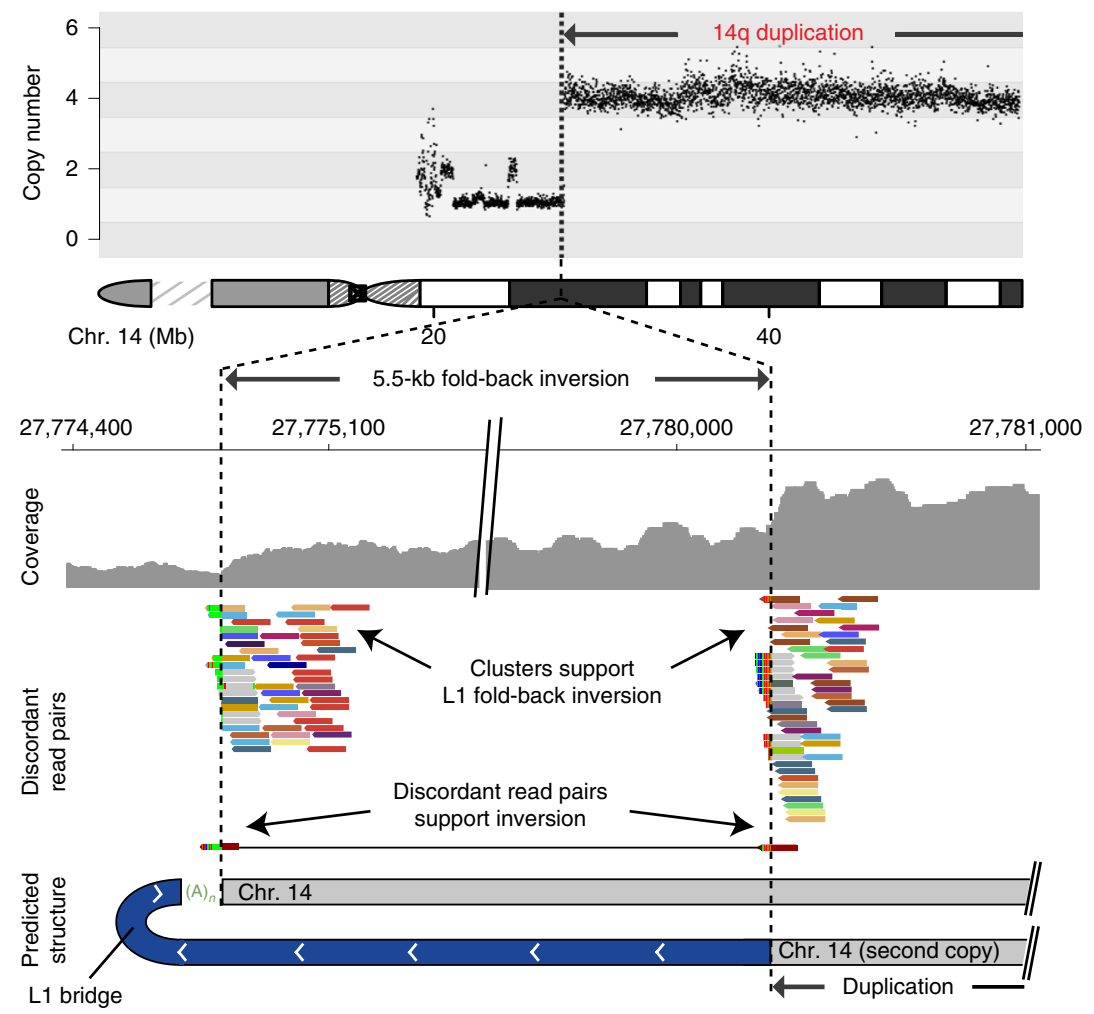

b

L1-mediated tandem duplication model
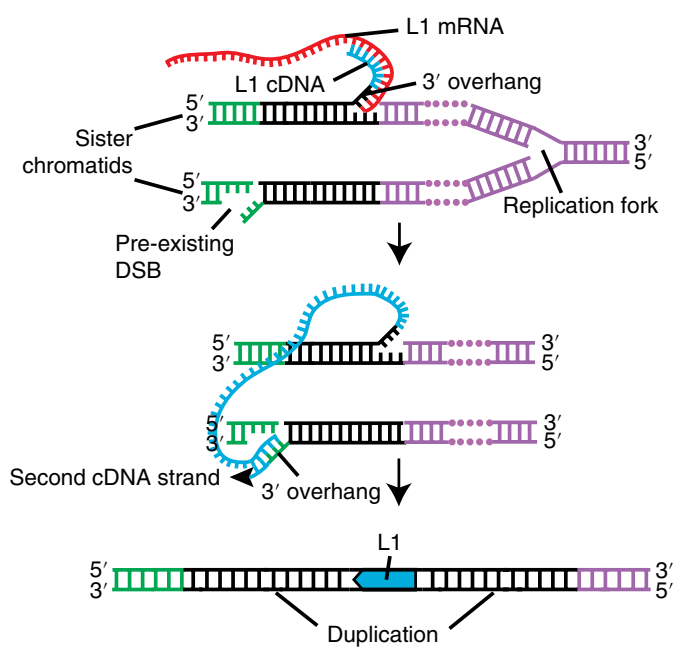

d L1-mediated foldback inversion model
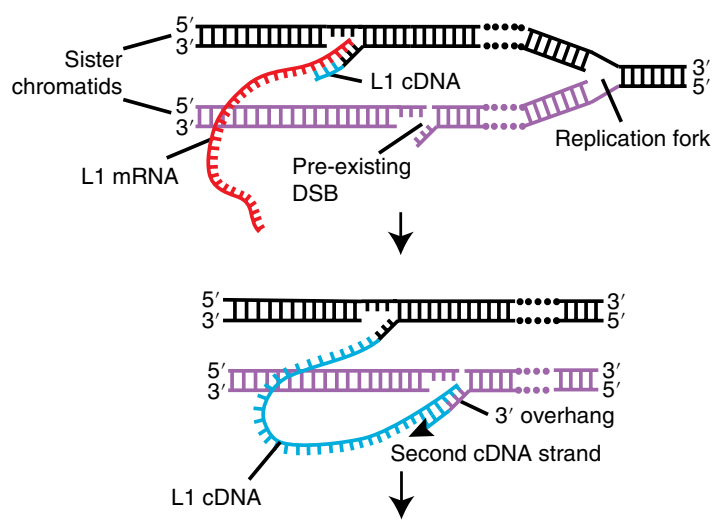

$\left.\right|_{\text {Duplication }} ^{5^{\prime}, \overline{1}}$ 


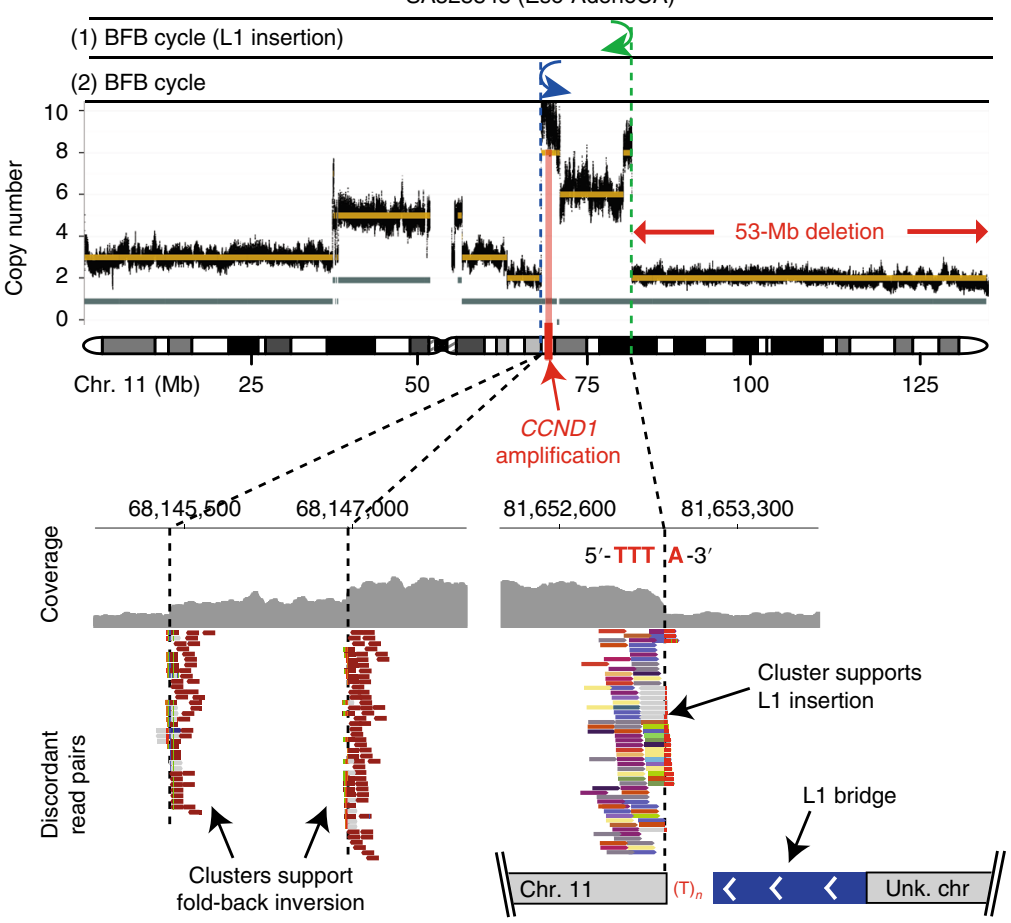

c

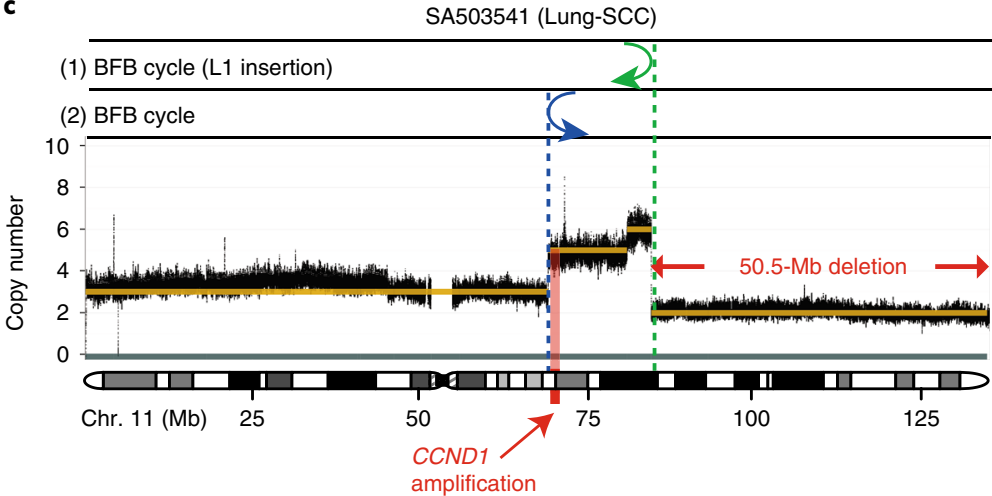

Fold-back inversion model

Original chromosome

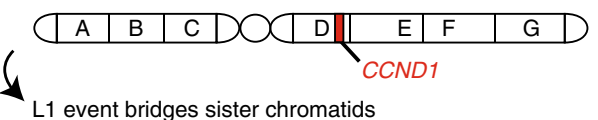

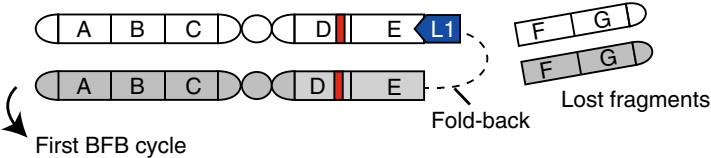

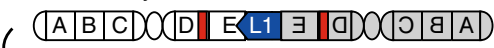

4

Poleward migration and breakage

4

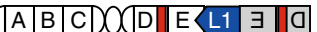

Anaphase bridge (second BFB)

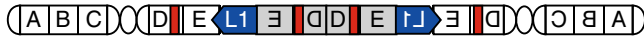

CCND1 amplification

Interchromosomal model

Original chromosomes

\begin{tabular}{|l|l|l|l|l||l|l|l|l|}
\hline $\mathrm{A}$ & $\mathrm{B}$ & $\mathrm{C}$ & $\mathrm{DO}$ & $\mathrm{D}$ & $\mathrm{E}$ & $\mathrm{F}$ & $\mathrm{G}$ & $\mathrm{D}$ \\
\hline
\end{tabular}

4

\begin{tabular}{|l|l|l|l|l|l|l|}
\hline & 1 & 2 & $D^{\prime}$ & 3 & 4 & 5 \\
\hline
\end{tabular}

L1 event bridges translocation

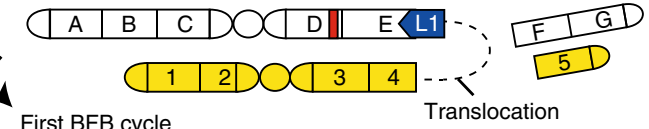

First BFB cycle

Translocation

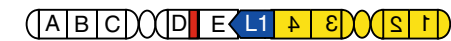

Poleward migration and breakage

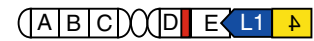

EDOCSTID

Anaphase bridge (second BFB)

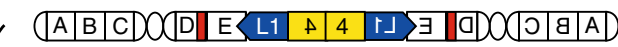

Poleward migration and breakage

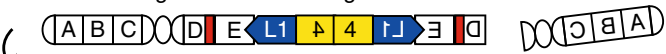

Anaphase bridge (third BFB)

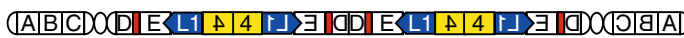

Fig. 8 | Somatic integration of L1 can trigger breakage-fusion-bridge cycles that lead to oncogene amplification. a, In esophageal adenocarcinoma sample SA528848, a single cluster of discordant reads (multi-colored reads) together with an L1-endonuclease cleavage site motif 5'-TTT/A-3' supports the integration of an $\mathrm{L} 1$ event that demarcates a 53-Mb telomeric (that is, including the telomere) deletion, from a region of massive amplification that involves CCND1. Around $14 \mathrm{Mb}$ upstream of the breakpoint of the deletion, we observed the presence of two clusters of read pairs (brown reads) that align close together and in the same orientation, which demarcate a change in copy number; this is a distinctive pattern of a fold-back inversion ${ }^{42,43}$, a rearrangement typically found to be associated with breakage-fusion-bridge (BFB) repair. In this fold-back inversion, the coverage shows much greater density on the right half of the rearrangement than the left, indicating that the abnormal chromosome is folded back on itself leading to duplicated genomic sequences in a head-to-head (inverted) orientation. The patterns described here suggest two independent breakage-fusion-bridge cycles, marked with (1) and (2). The copy-number plot shows the consensus total copy numbers (gold band) and the minor allele copy numbers (gray band). b. Models for the patterns described in $\mathbf{a}$. The fold-back inversion model involves two breakage-fusion-bridge cycles, one induced by L1-mediated fold-back inversion (see Fig. 7d), and a second induced by standard breakage-fusion-bridge repair. The interchromosomal rearrangement model involves an interchromosomal rearrangement mediated by an L1, followed by one extra cycle of breakage-fusion-bridge repair. c, In lung cancer sample SA503541, the integration of an $\mathrm{L} 1$ retrotransposon is associated with a 50-Mb loss on 11q that includes the telomere, and activates breakage-fusion-bridge repair, which leads to the amplification of CCND1.

can induce the first inverted rearrangement that generates end-to-end fusion of sister chromatids. In lung tumor sample SA313800 (Fig. 7c), we found a small L1 event inserted on chromosome $14 \mathrm{q}$ that demarcates a copy-number change that involves a $79.6-\mathrm{Mb}$ amplification of the $14 \mathrm{q}$ arm. Analysis of the sequencing data at the breakpoint revealed two discordant read clusters with the same orientation, which are $5.5 \mathrm{~kb}$ apart and support the integration of an L1. Both discordant clusters demarcate an increment of the sequencing coverage, for which the density is much greater in the right cluster. The only genomic structure that can explain this pattern is a fold-back inversion in which the two sister chromatids are bridged by an L1 retrotransposition in head-to-head (inverted) orientation (Fig. 7d).

In the example described above (Fig. 7c,d), no further breaks occurred, and the L1 retrotransposition generated an isochromosome (14q). In addition, we found examples in which the fusion of 
two chromatids by an L1 bridge induced further cycles of breakage-fusion-bridge repair. In esophageal tumor sample SA528848, we identified a cluster of reads on the long arm of chromosome 11 that had the typical hallmarks of an L1-mediated rearrangement (Fig. 8a). Copy-number data analysis showed that this L1 insertion point demarcated a 53-Mb deletion, which involved the loss of the telomeric region, from a region of massive amplification on chromosome 11. The amplified region on chromosome 11 contains the CCND1 oncogene, which is amplified in many human cancers ${ }^{41}$. The other end of this amplification was bound by a conventional fold-back inversion rearrangement (Fig. 8a), which is indicative of breakage-fusion-bridge repair ${ }^{42,43}$.

These patterns suggest the following sequence of events. During or soon after S phase, a somatic L1 retrotransposition bridges across sister chromatids in inverted orientation, breaking off the telomeric ends of 11q, which are then lost to the clone during the subsequent cell division (fold-back inversion model, Fig. 8b). The chromatids bridged by the L1 insertion now produce a dicentric chromosome. During mitosis, the two centromeres are pulled to opposite poles of the dividing cell, creating an anaphase bridge, which is resolved by further dsDNA breakage. This induces a second cycle of breakage-fusion-bridge repair, albeit not one mediated by L1 retrotransposition. These cycles lead to rapid-fire amplification of the CCND1 oncogene. Alternatively, an interchromosomal rearrangement mediated by L1 retrotransposition (interchromosomal rearrangement model, Fig. 8b) followed by two cycles of breakage-fusionbridge repair could generate similar copy-number patterns with telomere loss and amplification of CCND1.

Our data show that L1-mediated retrotransposition is an alternative mechanism of creating the first dicentric chromosome that induces subsequent rounds of chromosomal breakage and repair. If this occurs near an oncogene, the resulting amplification can provide a powerful selective advantage to the clone. We searched the PCAWG dataset for other rearrangements that included copy-number amplifications from telomeric deletions that were mediated by L1 integration. We found four more such events across three cancer samples (Supplementary Fig. 14). In a lung tumor sample, SA503541, we found almost identical rearrangements to the one described above (Fig. 8c). In this case, a somatic L1 event also generated telomere loss that induced a second cycle of breakage-fusion-bridge repair. The megabase-size amplification of chromosomal regions also targeted the CCND1 oncogene, in which the boundaries were demarcated by the L1 insertion breakpoint and a fold-back inversion, which indicates breakage-fusion-bridge repair. The independent occurrence of these patterns, which involve the amplification of CCND1, in two different tumor samples (SA528848 and SA503541) demonstrates a mutational mechanism mediated by L1 retrotransposition, which likely contributes to the development of human cancer.

\section{Discussion}

Here we characterize the patterns and mechanisms of cancer retrotransposition on a multidimensional scale, across 2,954 cancer genomes, integrated with rearrangement, transcriptomic and copy-number data. Our analyses provide a new perspective on the long-standing question of whether the activation of retrotransposons is relevant in human oncogenesis. Our findings demonstrate that major restructuring of cancer genomes can sometimes emerge from aberrant L1 retrotransposition events in tumors with high retrotransposition rates, particularly in esophageal, lung and head-and-neck cancers. L1-mediated deletions can promote the loss of megabase-scale regions of a chromosome that may involve centromeres and telomeres. It is likely that the majority of such genomic rearrangements would be harmful for a cancer clone. However, occasionally, L1-mediated deletions may promote cancer-driving rearrangements that involve the loss of tumor-suppressor genes and/or the amplification of oncogenes, representing another mechanism by which cancer clones acquire new mutations that help them to survive and grow. We expect that structural variants induced by somatic retrotransposition in human cancer are more frequent than we could unambiguously characterize here, given the constraints on the fragment sizes of paired-end sequencing libraries. Long-read sequencing technologies should be able to provide a more comprehensive picture of how frequent such events are. Relatively few germline L1 loci in a given tumor, typically one to three copies, are responsible for such marked structural remodeling. Given the role that these L1 copies may have in some cancer types, this work underscores the importance of characterizing cancer genomes for patterns of $\mathrm{L} 1$ retrotransposition.

\section{Online content}

Any methods, additional references, Nature Research reporting summaries, source data, extended data, supplementary information, acknowledgements, peer review information; details of author contributions and competing interests; and statements of data and code availability are available at https://doi.org/10.1038/ s41588-019-0562-0.

Received: 21 September 2017; Accepted: 26 November 2019; Published online: 5 February 2020

\section{References}

1. International Human Genome Sequencing Consortium Initial sequencing and analysis of the human genome. Nature 409, 860-921 (2001).

2. Kazazian, H. H. Jr. Mobile elements: drivers of genome evolution. Science 303, 1626-1632 (2004).

3. Sassaman, D. M. et al. Many human L1 elements are capable of retrotransposition. Nat. Genet. 16, 37-43 (1997).

4. Brouha, B. et al. Hot L1s account for the bulk of retrotransposition in the human population. Proc. Natl Acad. Sci. USA 100, 5280-5285 (2003).

5. Beck, C. R. et al. LINE-1 retrotransposition activity in human genomes. Cell 141, 1159-1170 (2010).

6. Menendez, L., Benigno, B. B. \& McDonald, J. F. L1 and HERV-W retrotransposons are hypomethylated in human ovarian carcinomas. Mol. Cancer 3, 12 (2004).

7. Tubio, J. M. et al. Extensive transduction of nonrepetitive DNA mediated by L1 retrotransposition in cancer genomes. Science 345, 1251343 (2014).

8. Holmes, S. E., Dombroski, B. A., Krebs, C. M., Boehm, C. D. \& Kazazian, H. H. Jr. A new retrotransposable human L1 element from the LRE2 locus on chromosome 1q produces a chimaeric insertion. Nat. Genet. 7, 143-148 (1994).

9. Moran, J. V., DeBerardinis, R. J. \& Kazazian, H. H. Jr. Exon shuffling by L1 retrotransposition. Science 283, 1530-1534 (1999).

10. Kazazian, H. H. Jr. Processed pseudogene insertions in somatic cells. Mob. DNA 5, 20 (2014).

11. Cooke, S. L. et al. Processed pseudogenes acquired somatically during cancer development. Nat. Commun. 5, 3644 (2014).

12. Ewing, A. D. et al. Retrotransposition of gene transcripts leads to structural variation in mammalian genomes. Genome Biol. 14, R22 (2013).

13. Lee, E. et al. Landscape of somatic retrotransposition in human cancers. Science 337, 967-971 (2012).

14. Helman, E. et al. Somatic retrotransposition in human cancer revealed by whole-genome and exome sequencing. Genome Res. 24, 1053-1063 (2014).

15. Solyom, S. et al. Extensive somatic L1 retrotransposition in colorectal tumors. Genome Res. 22, 2328-2338 (2012).

16. Symer, D. E. et al. Human L1 retrotransposition is associated with genetic instability in vivo. Cell 110, 327-338 (2002).

17. Gilbert, N., Lutz-Prigge, S. \& Moran, J. V. Genomic deletions created upon LINE-1 retrotransposition. Cell 110, 315-325 (2002).

18. Erwin, J. A. et al. L1-associated genomic regions are deleted in somatic cells of the healthy human brain. Nat. Neurosci. 19, 1583-1591 (2016).

19. Burns, K. H. Transposable elements in cancer. Nat. Rev. Cancer 17, 415-424 (2017).

20. Kazazian, H. H. Jr. \& Moran, J. V. Mobile DNA in health and disease. N. Engl. J. Med 377, 361-370 (2017).

21. The ICGC/TCGA Pan-Cancer Analysis of Whole Genomes Consortium. Pan-cancer analysis of whole genomes. Nature https://doi.org/10.1038/ s41586-020-1969-6 (2020).

22. Wylie, A. et al. p53 genes function to restrain mobile elements. Genes Dev. 30, 64-77 (2016). 
23. Jung, H., Choi, J. K. \& Lee, E. A. Immune signatures correlate with L1 retrotransposition in gastrointestinal cancers. Genome Res. 28, 1136-1146 (2018).

24. Supek, F. \& Lehner, B. Clustered mutation signatures reveal that error-prone DNA repair targets mutations to active genes. Cell 170, 534-547 (2017)

25. Mita, P. et al. LINE-1 protein localization and functional dynamics during the cell cycle. eLife 7, e30058 (2018).

26. Barski, A. et al. High-resolution profiling of histone methylations in the human genome. Cell 129, 823-837 (2007).

27. Kimberland, M. L. et al. Full-length human L1 insertions retain the capacity for high frequency retrotransposition in cultured cells. Hum. Mol. Genet 8, 1557-1560 (1999).

28. Chang, H. H. Y., Pannunzio, N. R., Adachi, N. \& Lieber, M. R. Non-homologous DNA end joining and alternative pathways to double-strand break repair. Nat. Rev. Mol. Cell Biol. 18, 495-506 (2017).

29. Han, K. et al. Genomic rearrangements by LINE-1 insertion-mediated deletion in the human and chimpanzee lineages. Nucleic Acids Res. 33, 4040-4052 (2005).

30. Sen, S. K., Huang, C. T., Han, K. \& Batzer, M. A. Endonuclease-independent insertion provides an alternative pathway for $\mathrm{L} 1$ retrotransposition in the human genome. Nucleic Acids Res. 35, 3741-3751 (2007).

31. Farkash, E. A., Kao, G. D., Horman, S. R. \& Prak, E. T. Gamma radiation increases endonuclease-dependent L1 retrotransposition in a cultured cell assay. Nucleic Acids Res. 34, 1196-1204 (2006).

32. Gilbert, N., Lutz, S., Morrish, T. A. \& Moran, J. V. Multiple fates of L1 retrotransposition intermediates in cultured human cells. Mol. Cell Biol. 25, 7780-7795 (2005)

33. Zhou, C., Li, J. \& Li, Q. CDKN2A methylation in esophageal cancer: a meta-analysis. Oncotarget 8, 50071-50083 (2017).

34. The Cancer Genome Atlas Research Network Comprehensive genomic characterization of squamous cell lung cancers. Nature 489, 519-525 (2012).

35. The Cancer Genome Atlas Network Comprehensive genomic characterization of head and neck squamous cell carcinomas. Nature 517, 576-582 (2015).

36. The Cancer Genome Atlas Research Network Comprehensive molecular profiling of lung adenocarcinoma. Nature 511, 543-550 (2014).

37. $\mathrm{Xu}, \mathrm{W}$. \& Ji, J. Y. Dysregulation of CDK8 and cyclin C in tumorigenesis. J. Genet. Genomics 38, 439-452 (2011).

38. Artandi, S. E. et al. Telomere dysfunction promotes non-reciprocal translocations and epithelial cancers in mice. Nature 406 641-645 (2000).

39. O’Hagan, R. C. et al. Telomere dysfunction provokes regional amplification and deletion in cancer genomes. Cancer Cell 2, 149-155 (2002).

40. Maciejowski, J. \& de Lange, T. Telomeres in cancer: tumour suppression and genome instability. Nat. Rev. Mol. Cell Biol. 18, 175-186 (2017).

41. Beroukhim, R. et al. The landscape of somatic copy-number alteration across human cancers. Nature 463, 899-905 (2010).

42. Campbell, P. J. et al. The patterns and dynamics of genomic instability in metastatic pancreatic cancer. Nature 467, 1109-1113 (2010).

43. Li, Y. et al. Constitutional and somatic rearrangement of chromosome 21 in acute lymphoblastic leukaemia. Nature 508, 98-102 (2014).

Publisher's note Springer Nature remains neutral with regard to jurisdictional claims in published maps and institutional affiliations.

Open Access This article is licensed under a Creative Commons
Attribution 4.0 International License, which permits use, sharing, adap-
tation, distribution and reproduction in any medium or format, as long
as you give appropriate credit to the original author(s) and the source, provide a link to
the Creative Commons license, and indicate if changes were made. The images or other
third party material in this article are included in the article's Creative Commons license,
unless indicated otherwise in a credit line to the material. If material is not included in
the article's Creative Commons license and your intended use is not permitted by statu-
tory regulation or exceeds the permitted use, you will need to obtain permission directly
from the copyright holder. To view a copy of this license, visit http://creativecommons.
org/licenses/by/4.0/.
(c) The Author(s) 2020

'Department of Zoology, Genetics and Physical Anthropology, Universidade de Santiago de Compostela, Santiago de Compostela, Spain. ${ }^{2}$ Biomedical Research Centre (CINBIO), University of Vigo, Vigo, Spain. ${ }^{3}$ Centre for Research in Molecular Medicine and Chronic Diseases (CIMUS), Universidade de Santiago de Compostela, Santiago de Compostela, Spain. ${ }^{4}$ Transmissible Cancer Group, Department of Veterinary Medicine, University of Cambridge, Cambridge, UK. ${ }^{5}$ The Biomedical Research Centre (CINBIO), Universidade de Vigo, Vigo, Spain. ${ }^{6}$ Wellcome Sanger Institute, Wellcome Genome Campus, Cambridge, UK. ${ }^{7}$ Genome Data Science, Institute for Research in Biomedicine (IRB Barcelona), The Barcelona Institute of Science and Technology (BIST), Barcelona, Spain. ${ }^{8}$ Institucio Catalana de Recerca i Estudis Avançats (ICREA), Barcelona, Spain. ${ }^{9}$ The Francis Crick Institute, London, UK. ${ }^{10}$ Department of Human Genetics, University of Leuven, Leuven, Belgium. ${ }^{11}$ Genomes and Disease, Centre for Research in Molecular Medicine and Chronic Diseases (CIMUS), Universidade de Santiago de Compostela, Santiago de Compostela, Spain. ${ }^{12}$ Graduate School of Medical Science and Engineering, Korea Advanced Institute of Science and Technology, Daejeon, South Korea. ${ }^{13}$ Cancer Ageing and Somatic Mutation Programme, Wellcome Sanger Institute, Cambridge, UK. ${ }^{14}$ Department of Biochemistry, Genetics and Immunology, University of Vigo, Vigo, Spain. ${ }^{15}$ Galicia Sur Health Research Institute, Vigo, Spain. ${ }^{16}$ Faculty of Science and Technology, University of Vic-Central University of Catalonia (UVic-UCC), Vic, Spain. ${ }^{17}$ Barcelona Supercomputing Center (BSC), Barcelona, Spain. ${ }^{18}$ Experimental Cancer Genetics, Wellcome Sanger Institute, Cambridge, UK. ${ }^{19}$ Oxford Big Data Institute, University of Oxford, Oxford, UK. ${ }^{20}$ DNA Repair and Genome Integrity, Centre for Research in Molecular Medicine and Chronic Diseases (CIMUS), Universidade de Santiago de Compostela, Santiago de Compostela, Spain. ${ }^{21}$ Department of Biochemistry and Molecular Biology, Universidade de Santiago de Compostela, Santiago de Compostela, Spain. ${ }^{22}$ Medical Research Council (MRC) Cancer Unit, University of Cambridge, Cambridge, UK. ${ }^{23}$ Department of Genetic Medicine, Johns Hopkins University School of Medicine, Baltimore, Baltimore, MD, USA. ${ }^{24}$ University of Cambridge, Cambridge, UK. ${ }^{25}$ Li Ka Shing Centre, Cancer Research UK Cambridge Institute, University of Cambridge, Cambridge, UK. ${ }^{26}$ European Molecular Biology Laboratory (EMBL), Genome Biology Unit, Heidelberg, Germany. ${ }^{27}$ Finsen Laboratory and Biotech Research \& Innovation Centre (BRIC), University of Copenhagen, Copenhagen, Denmark. ${ }^{28}$ Department of Urology, Charité Universitätsmedizin Berlin, Berlin, Germany. ${ }^{29}$ Department of Bioinformatics and Computational Biology, The University of Texas MD Anderson Cancer Center, Houston, TX, USA. ${ }^{30}$ Department of Genetics and Informatics Institute, University of Alabama at Birmingham (UAB) School of Medicine, Birmingham, AL, USA. ${ }^{31}$ University of Texas MD Anderson Cancer Center, Houston, TX, USA. ${ }^{32}$ Division of Genetics and Genomics, Boston Children's Hospital, Harvard Medical School, Boston, MA, USA. ${ }^{33}$ The Broad Institute of Harvard and MIT, Cambridge, MA, USA. ${ }^{34}$ Department of Cancer Biology, Dana-Farber Cancer Institute, Boston, MA, USA. ${ }^{35}$ Department of Medical Oncology, Dana-Farber Cancer Institute, Boston, MA, USA. ${ }^{36}$ Harvard Medical School, Boston, MA, USA. ${ }^{37}$ Program in Computational Biology and Bioinformatics, Yale University, New Haven, CT, USA. ${ }^{38}$ Department of Molecular Biophysics and Biochemistry, Yale University, New Haven, CT, USA. ${ }^{39}$ Department of Computer Science, Yale University, New Haven, CT, USA. ${ }^{40}$ Department of Human Genetics, McGill University, Montreal, Québec, Canada. ${ }^{41}$ Department of Oncology and OncoHematology, University of Milan, Milan, Italy. ${ }^{42}$ Department of Medical Oncology and Hematology, Fondazione IRCCS Istituto Nazionale dei Tumori, Milan, Italy. ${ }^{43}$ Canadian Center for Computational Genomics, McGill University, Montreal, Quebec, Canada. ${ }^{44}$ Department of Biomedical Informatics, Harvard Medical School, Boston, MA, USA. ${ }^{45}$ Ludwig Center at Harvard, Boston, MA, USA. ${ }^{46}$ Oxford NIHR Biomedical Research Centre, Oxford, UK. ${ }^{47}$ European Molecular Biology Laboratory, European Bioinformatics Institute (EMBL-EBI), Cambridge, UK. ${ }^{48}$ Department of Pathology, Johns Hopkins University School of Medicine, Baltimore, Baltimore, MD, USA. ${ }^{49}$ McKusick-Nathans Institute of Genetic Medicine, Sidney Kimmel Comprehensive Cancer Center at Johns Hopkins University School of Medicine, Baltimore, MD, USA. ${ }^{50} \mathrm{~A}$ full list of members and their affiliations appears at the end of the paper. ${ }^{51}$ Department of Haematology, University of Cambridge, Cambridge, UK. ${ }^{52} \mathrm{~A}$ full list of members and their affiliations appears online. ${ }^{920}$ These authors contributed equally: Eva G. Alvarez, Adrian Baez-Ortega, Jorge Zamora, Fran Supek. ${ }^{921}$ These authors jointly supervised this work: Peter J. Campbell, Jose M. C. Tubio.凶e-mail: pc8@sanger.ac.uk; jose.mc.tubio@usc.es 


\section{Kadir C. Akdemir ${ }^{31}$, Eva G. Alvarez 1,2,3, Adrian Baez-Ortega ${ }^{4}$, Rameen Beroukhim ${ }^{33,35,36}$, Paul C. Boutros ${ }^{53,54,55,56}$, David D. L. Bowtell ${ }^{57,58}$, Benedikt Brors ${ }^{59,60,61}$, Kathleen H. Burns ${ }^{48,49}$, Peter J. Campbell ${ }^{6,51}$, Kin Chan ${ }^{62}$, Ken Chen ${ }^{31}$, Isidro Cortés-Ciriano ${ }^{44,63,64}$, Ana Dueso-Barroso ${ }^{16,17}$, Andrew J. Dunford ${ }^{65}$, Paul A. Edwards ${ }^{24,25}$, Xavier Estivill66, Dariush Etemadmoghadam57,67, Lars Feuerbach $^{59}$, J. Lynn Fink ${ }^{17,68}$, Milana Frenkel-Morgenstern ${ }^{69}$, Dale W. Garsed ${ }^{57,67}$, Mark Gerstein ${ }^{37,38,39}$, Dmitry A. Gordenin ${ }^{70}$, David Haan ${ }^{71}$, James E. Haber ${ }^{72}$, Julian M. Hess ${ }^{65,73}$, Barbara Hutter ${ }^{61,74,75}$, Marcin Imielinski ${ }^{76,77}$, David T. W. Jones ${ }^{78,79}$, Young Seok Ju ${ }^{6,80}$, Marat D. Kazanov ${ }^{81,82,83}$, Leszek J. Klimczak ${ }^{84}$, Youngil Koh ${ }^{85,86}$, Jan O. Korbel ${ }^{26,47}$, Kiran Kumar ${ }^{65}$, Eunjung Alice Lee ${ }^{32}$, Jake June-Koo Lee ${ }^{44,45}$, Yilong Li ${ }^{6}$, Andy G. Lynch ${ }^{24,87,88,89}$, Geoff Macintyre ${ }^{87}$, Florian Markowetz ${ }^{24,87}$, Iñigo Martincorena ${ }^{6}$, Alexander Martinez-Fundichely ${ }^{90,91,92}$, Matthew Meyerson 36,65,93,94,95, Satoru Miyano96, Hidewaki Nakagawa97, Fabio C. P. Navarro37,38,39, Stephan Ossowski ${ }^{98,99,100}$, Peter J. Park ${ }^{44,45}$, John V. Pearson ${ }^{101,102}$, Montserrat Puiggròs ${ }^{17}$, Karsten Rippe ${ }^{103}$, Nicola D. Roberts ${ }^{6}$, Steven A. Roberts ${ }^{104}$, Bernardo Rodriguez-Martin ${ }^{1,2,3}$, Steven E. Schumacher ${ }^{33,34,35}$, Ralph Scully ${ }^{105}$, Mark Shackleton ${ }^{66,106}$, Nikos Sidiropoulos ${ }^{27}$, Lina Sieverling ${ }^{59,107}$, Chip Stewart ${ }^{65}$, David Torrents ${ }^{8,17}$, Jose M. C. Tubio ${ }^{1,2,3,13}$, Izar Villasante ${ }^{17}$, Nicola Waddell ${ }^{100,101}$, Jeremiah A. Wala33,34,35,36, Joachim Weischenfeldt ${ }^{26,27,28}$, Lixing Yang ${ }^{108}$, Xiaotong Yao ${ }^{75,109}$, Sung-Soo Yoon ${ }^{85}$, Jorge Zamora ${ }^{1,3,5,6}$ and Cheng-Zhong Zhang ${ }^{36,65,92}$}

\footnotetext{
${ }^{53}$ Computational Biology Program, Ontario Institute for Cancer Research, Toronto, Ontario, Canada. ${ }^{54}$ Department of Medical Biophysics, University of Toronto, Toronto, Ontario, Canada. ${ }^{55}$ Department of Pharmacology, University of Toronto, Toronto, Ontario, Canada. ${ }^{56}$ University of California Los Angeles, Los Angeles, CA, USA. ${ }^{57}$ Peter MacCallum Cancer Centre, Melbourne, Victoria, Australia. ${ }^{58}$ Sir Peter MacCallum Department of Oncology, University of Melbourne, Melbourne, Victoria, Australia. ${ }^{59}$ National Center for Tumor Diseases (NCT) Heidelberg, Heidelberg, Germany. ${ }^{60}$ Johns Hopkins School of Medicine, Baltimore, MD, USA. ${ }^{6}$ Division of Applied Bioinformatics, German Cancer Research Center (DKFZ), Heidelberg, Germany. ${ }^{62}$ Department of Biochemistry, Microbiology and Immunology, Faculty of Medicine, University of Ottawa, Ottawa, Ontario, Canada. ${ }^{63}$ Centre for Molecular Science Informatics, Department of Chemistry, University of Cambridge, Cambridge, UK. ${ }^{64}$ Ludwig Center, Harvard Medical School, Boston, MA, USA. ${ }^{65}$ Broad Institute of MIT and Harvard, Cambridge, MA, USA. ${ }^{66}$ Sidra Medicine, Doha, Qatar. ${ }^{67}$ Sir Peter MacCallum Department of Oncology, The University of Melbourne, Melbourne, Victoria, Australia. ${ }^{68}$ Queensland Centre for Medical Genomics, Institute for Molecular Bioscience, The University of Queensland, St Lucia, Queensland, Australia. ${ }^{69}$ The Azrieli Faculty of Medicine, Bar-Ilan University, Safed, Israel. ${ }^{70}$ Genome Integrity and Structural Biology Laboratory, National Institute of Environmental Health Sciences (NIEHS), Durham, NC, USA. ${ }^{71 B i o m o l e c u l a r ~ E n g i n e e r i n g ~ D e p a r t m e n t, ~ U n i v e r s i t y ~ o f ~ C a l i f o r n i a, ~ S a n t a ~}$ Cruz, Santa Cruz, CA, USA. ${ }^{72}$ Brandeis University, Waltham, MA, USA. ${ }^{73}$ Massachusetts General Hospital Center for Cancer Research, Charlestown, MA, USA. ${ }^{74}$ German Cancer Consortium (DKTK), Heidelberg, Germany. ${ }^{75}$ Heidelberg Center for Personalized Oncology (DKFZ-HIPO), German Cancer Research Center (DKFZ), Heidelberg, Germany. ${ }^{76} \mathrm{New}$ York Genome Center, New York, NY, USA. ${ }^{77}$ Weill Cornell Medicine, New York, NY, USA. ${ }^{78} \mathrm{Hopp}$ Children's Cancer Center (KiTZ), Heidelberg, Germany. ${ }^{79}$ Pediatric Glioma Research Group, German Cancer Research Center (DKFZ), Heidelberg, Germany. ${ }^{80}$ Korea Advanced Institute of Science and Technology, Daejeon, South Korea. ${ }^{81}$ Skolkovo Institute of Science and Technology, Moscow, Russia. ${ }^{82} \mathrm{~A}$. A. Kharkevich Institute of Information Transmission Problems, Moscow, Russia. ${ }^{83}$ Dmitry Rogachev National Research Center of Pediatric Hematology, Oncology and Immunology, Moscow, Russia. ${ }^{84}$ Integrative Bioinformatics Support Group, National Institute of Environmental Health Sciences (NIEHS), Durham, NC, USA. ${ }^{85}$ Center For Medical Innovation, Seoul National University Hospital, Seoul, South Korea. ${ }^{86}$ Department of Internal Medicine, Seoul National University Hospital, Seoul, South Korea. ${ }^{87}$ Cancer Research UK Cambridge Institute, University of Cambridge, Cambridge, UK. ${ }^{88}$ School of Medicine, University of St Andrews, St Andrews, UK. ${ }^{89}$ School of Mathematics and Statistics, University of St Andrews, St Andrews, UK. ${ }^{90}$ Department of Physiology and Biophysics, Weill Cornell Medicine, New York, NY, USA. ${ }^{91}$ Englander Institute for Precision Medicine, Weill Cornell Medicine, New York, NY, USA. ${ }^{92}$ Institute for Computational Biomedicine, Weill Cornell Medicine, New York, NY, USA. ${ }^{93}$ Dana-Farber Cancer Institute, Boston, MA, USA. ${ }^{94}$ Department of Medical Oncology, Inselspital, University Hospital and University of Bern, Bern, Switzerland. ${ }^{95}$ Department of Pathology, The University of Melbourne, Melbourne, Victoria, Australia. ${ }^{96}$ The Institute of Medical Science, The University of Tokyo, Tokyo, Japan. ${ }^{97}$ RIKEN Center for Integrative Medical Sciences, Yokohama, Japan. ${ }^{98}$ Centre for Genomic Regulation (CRG), The Barcelona Institute of Science and Technology, Barcelona, Spain. ${ }^{99}$ Institute of Medical Genetics and Applied Genomics, University of Tübingen, Tübingen, Germany. ${ }^{100}$ Universitat Pompeu Fabra (UPF), Barcelona, Spain. ${ }^{101}$ Department of Genetics and Computational Biology, QIMR Berghofer Medical Research Institute, Brisbane, Australia. ${ }^{102}$ Institute for Molecular Bioscience, University of Queensland, St Lucia, Brisbane, Queensland, Australia. ${ }^{103}$ German Cancer Research Center (DKFZ), Heidelberg, Germany. ${ }^{104}$ School of Molecular Biosciences and Center for Reproductive Biology, Washington State University, Pullman, WA, USA. ${ }^{105}$ Cancer Research Institute, Beth Israel Deaconess Medical Center, Boston, MA, USA. ${ }^{106}$ Peter MacCallum Cancer Centre and University of Melbourne, Melbourne, Victoria, Australia. ${ }^{107}$ Faculty of Biosciences, Heidelberg University, Heidelberg, Germany. ${ }^{108}$ Ben May Department for Cancer Research, Department of Human Genetics, The University of Chicago, Chicago, IL, USA. ${ }^{109}$ Tri-institutional PhD Program of Computational Biology and Medicine, Weill Cornell Medicine, New York, NY, USA.
} 


\section{Methods}

Pan-cancer datasets. Whole-genome sequencing dataset. We analyzed Illumina whole-genome paired-end sequencing reads ( $100-150 \mathrm{bp})$ from 2,954 tumors and matched normal samples across 38 cancer types $^{21}$. On the basis of the robustness of the retrotransposition calls (false discovery rate of $<5 \%$ ), we opted to retain all samples that were preliminarily excluded by the PCAWG Consortium ${ }^{21}$, as they were excluded from SNV and structural variation analyses on the basis of read direction biases from PCR artifacts or poor sequence quality, but were not found to be problematic for retrotransposition analysis. For the majority of donors, the tumor specimens consisted of a fresh frozen sample, whereas the normal specimens consisted of a blood sample. Most of the tumor samples came from treatment-free primary cancers, although there was also a small number of donors with multiple samples of primary, metastatic and/or recurrent tumors. The average coverage was 30 reads per genome for normal samples, whereas tumor samples had a bimodal coverage distribution with maxima at 38 and 60 reads per bp (Supplementary Fig. 1 and Supplementary Table 1). BWA-mem ${ }^{44}$ v.0.7.8-r455 was used to align each tumor and normal sample to human reference build GRCh37. Additional technical details of the sequencing metrics are provided in Supplementary Table 1 and in the PCAWG lead paper ${ }^{21}$. The Ethics oversight for the PCAWG protocol was undertaken by the TCGA Program Office and the Ethics and Governance Committee of the ICGC.

Transcriptome dataset. About half of the donors $(1,188)$ with whole-genome data in PCAWG had at least one tumor specimen with whole transcriptome obtained by RNA sequencing (RNA-seq). Mapping onto the reference was carried out using two independent read aligners, STAR ${ }^{45}$ v.2.4.0i, two-pass and TopHat2 (ref. ${ }^{46}$ ) v.2.0.12. Gene expression was quantified with $\mathrm{HTSeq}^{47} \mathrm{v} \cdot 0.6 .1 \mathrm{p} 1$ and consensus normalized expression values, in fragments per kilobase of transcript per million mapped reads (FPKM), were obtained by averaging the expression from STAR and TopHat2. A more detailed description of RNA-seq data processing is provided by the PCAWG Integration of Transcriptome and Genome Working Group ${ }^{48}$.

Copy-number dataset. We analyzed copy-number profiles obtained by the PCAWG Evolution and Heterogeneity Working Group, using a consensus approach that combines six different state-of-the-art copy-number calling methods ${ }^{49}$. GC content corrected $\operatorname{logR}$ values were extracted using the Battenberg algorithm ${ }^{50}$, smoothed using a running median and transformed into copy-number space according to $n=\left[(2(1-\rho)+\psi \rho) 2^{\operatorname{logR}}-2(1-\rho)\right] / \rho$ where $\rho$ and $\psi$ are consensus tumor purity and ploidy, respectively.

Structural variant dataset. The structural variation call set was generated by the PCAWG Somatic Structural Variation Working Group by merging the structural variant calls from four independent calling pipelines ${ }^{51}$. The merged structural variant calls were further required to have a consistent change in copy number.

Analysis of somatic retrotransposition. Detection of mobile element insertions using TraFiC-mem. BAM files from tumor and matched normal pairs were processed with TraFiC-mem v.1.1.0 to identify somatic mobile element insertions (MEIs) including solo-L1, L1-mediated transductions, Alu, SVA and ERV-K using Illumina paired-end mapping data. TraFiC-mem starts by identifying candidate somatic MEIs by analyzing discordant read pairs. In contrast to a previous version of the algorithm ${ }^{7}$, the new pipeline uses BWA-mem v.0.7.17 instead of RepeatMasker as the search engine for the identification of retrotransposon-like sequences in the sequencing reads. Calls obtained at this step are preliminary, in which MEI features are outlined and insertion coordinates represent ranges that surround the breakpoints. Then, a new module of TraFiC-mem, called MEIBA (from Mobile Element Insertion Breakpoint Analyzer), is used to identify the integration breakpoints to base-pair resolution and to perform a detailed characterization of MEI features, including structure, subfamily assignment and insertion site annotation. TraFiC-mem is illustrated in Supplementary Fig. 3. Detailed information about the pipeline is provided in the Supplementary Note.

Identification of germline and somatic L1 source elements. Because L1-mediated transductions are defined by the retrotransposition of unique, non-repetitive genomic sequences, we can unambiguously identify the L1 source element from which they derive? The method relies on the detection of unique DNA regions retrotransposed somatically elsewhere in the cancer genome from a single locus that matches the $10-\mathrm{kb}$ downstream region of a reference full-length L1 element or a putative non-reference polymorphic L1 element detected by TraFiC-mem across the matched normal samples in the PCAWG cohort ${ }^{21}$. When transduced regions were derived from the downstream region of a putative L1 event present in the tumor genome but not in the matched normal genome, we catalogued these elements as somatic L1 source loci.

Identification of processed pseudogene insertions. An additional separate module of TraFiC-mem was implemented for the identification of somatic insertions of processed pseudogenes. The method relies on the same principle as for the identification of somatic MEI events, through the detection of two reciprocal clusters of discordant read pairs, namely positive and negative, that supports an insertion event in the reference genome. However, the method differs from standard MEI calling to which the read mates map, as in this case mates are required to map to exons that belong to the same source protein-coding gene in GENCODE v.19. To avoid misclassification with standard genomic rearrangements that involve coding regions, we use MEIBA-described above-to reconstruct the insertion breakpoint junctions looking for hallmarks of retrotransposition, including the poly $(\mathrm{A})$ tract and duplication of the target site. Candidate insertions without a $\operatorname{poly}(\mathrm{A})$ tail were discarded.

Identification of L1-mediated deletions. Independent read clusters, identified with TraFic-mem, supporting an L1 event (that is, clusters of discordant read pairs with no apparent reciprocal cluster within the proximal $500 \mathrm{bp}$, and for which the mates support a somatic L1 retrotransposition event) were interrogated for the presence of an associated change in copy number in its proximity. In brief, we looked for copy-number loss calls from the PCAWG Evolution and Heterogeneity Working Group for which the following conditions were fulfilled: (1) the upstream breakpoint matches an independent L1 cluster in positive orientation, (2) the corresponding downstream breakpoint, if any, from the same change in copy number matches an independent L1 cluster in negative orientation, and (3) the reconstruction of the structure of the putative insertion causing the deletion is compatible with one-single retrotransposition event. We used MEIBA (Supplementary Note) to reconstruct the insertion breakpoint junctions to confirm the ends of the events and identify hallmarks of retrotransposition, including the poly(A) tract and duplication of the target site.

An additional strategy was used for L1-mediated deletions that were shorter than $100 \mathrm{~kb}$. Read-depth drops in the proximity of independent clusters were detected by, first, normalizing the read depth on each side of the cluster, using the matched normal sample as a reference. Then, the ratio between the normalized read depth on both sides of the cluster was computed for windows of 200-5,000 bp. Adjacent buffer regions of $300 \mathrm{bp}$ on each side of the cluster were omitted from read-depth calculations to avoid false positives caused by sequence repeats. Pairs of independent reciprocal (positive-negative) clusters were selected such that: (1) both clusters were located less than $100 \mathrm{~kb}$ apart, (2) a potential drop in the read-depth ratio was identified, extending from the positive cluster to the negative cluster, and (3) the reconstruction of the structure of the putative insertion that caused the deletion was compatible with a single L1 event. For each cluster pair, the continuity and reliability of the copy-number drop was assessed by measuring the normalized read-depth ratio between non-overlapping 500-bp windows that spanned the region between the positive and negative clusters (that is the putative deletion) and windows upstream and downstream of the positive and negative clusters, respectively. The significance of each read-depth ratio drop was estimated nonparametrically using a null distribution of normalized read-depth ratios. This distribution was obtained for each tumor sample by randomly sampling 100,000 genomic locations (from copy-number segments showing the predominant copy number), and calculating read-depth ratios between both sides of each position. Nonparametric $P$ values were calculated by comparing observed read-depth ratios with this null distribution, and adjusted using the Benjamini-Hochberg correction. Two cluster groups were produced: tier 1, pairs of reciprocal clusters with both clusters that had $P<0.1$, and tier 2, pairs of reciprocal clusters with only one of both clusters having $P<0.1$.

Retrotransposition rate enrichment and depletion across tumor types. For each tumor type with a minimum sample size of 15 , we assessed whether it was enriched or depleted in retrotransposition compared to the overall retrotransposition burden using zero-inflated negative binomial regression, as implemented in the zeroinfl function of the pscl R package. This type of model takes into account the excess of zeros and the overdispersion that is present in this dataset. The MEI counts per sample were regressed on a binary factor that expressed whether they belonged to that particular type of cancer or to any other cancer type. On each regression, the magnitude and sign of the $z$-score indicates the effect size and directionality of the association. More specifically, positive $z$-scores indicate that a higher number of counts in the samples belongs to a particular cancer type compared with the rest (enrichment), whereas negative scores indicate a lower number of counts (depletion). Each $z$-score is accompanied by its $P$ value to indicate the level of statistical significance.

Association between mutation in tumor-suppressor genes and retrotransposition and structural variantion rates. To assess whether the disruption of a particular tumor-suppressor gene was associated with a high level of retrotransposition, we used the whole-genome panorama of cancer driver events per sample produced by the PCAWG Drivers and Functional Interpretation Working Group ${ }^{21}$. This panorama includes coding and non-coding SNVs, insertions and deletions, copy-number alterations, structural variants and potentially predisposing germline variants. For each tumor-suppressor gene in the Cancer Gene Census database with mutational data, we stratified the samples into two groups-mutated tumor-suppressor genes and non-mutated tumor-suppressor genes. Then, we compared the distribution of MEI counts between both groups using a MannWhitney $U$-test to identify significant differences. $P$ values were corrected for multiple testing using the Benjamini-Hochberg procedure. Adjusted $P<0.05$ were considered significant. This analysis was done at both the level of the 
individual cancer type and the level of pan-cancer to identify tumor-type-specific associations. We further investigated whether there was a TP53 dosage effect as follows: every PCAWG sample was classified into three groups according to TP53 mutational status, namely wild-type, monoallelic and biallelic driver mutation. Then, the MEI counts distribution was compared for all possible group pair combinations using a Mann-Whitney $U$-test. The same analysis described above was applied to investigate the association between TP53 mutation and other types of structural variation.

Correlation between $L 1$ insertion and structural variation rate. For each sample, we computed the number of MEIs, the total number of structural variants and the number of five different structural variant classes: deletions, duplications, translocations, head-to-head inversions and tail-to-tail inversions, when data were available. Then, the correlation between the number of MEIs and the structural variant burden was assessed at both the level of the individual tumor type and the level of the pan-cancer using a Spearman's rank test.

Association between $\mathrm{L} 1$ insertion rate and genomic features. The L1 insertion rate was calculated as the total number of somatic L1 insertions, identified across the complete PCAWG cohort per $1-\mathrm{Mb}$ window. The density of $\mathrm{L} 1$ endonuclease motifs was computed as the number of canonical endonuclease motifs, here defined as TTTT|R (where $\mathrm{R}$ is $\mathrm{A}$ or $\mathrm{G}$ ) or $\mathrm{Y} \mid \mathrm{AAAA}$ (where $\mathrm{Y}$ is $\mathrm{C}$ or $\mathrm{T}$ ) per 1-Mb window. To study the association of $\mathrm{L} 1$ insertion rate with multiple variables at single-nucleotide resolution, we used a statistical framework based on negative binomial regression, as described in detail previously ${ }^{24}$, and adapted to adjust for the genome-wide distribution of the L1 endonuclease motif; we stratified the genome into four bins (0-3) by the closeness of match to the motif. Bin 0 contains dissimilar DNA motifs, with four or five (out of five) mismatches, encompassing 1,150 Mb. Bins 1,2 and 3 contain loci with three, two and at most one mismatches, encompassing $749 \mathrm{Mb}$, $380 \mathrm{Mb}$ and $114 \mathrm{Mb}$, respectively. The closer match of either of the two DNA strands at each locus was considered. Histone mark data and DNase hypersensitivity data were obtained from the Roadmap Epigenomics Consortium by averaging the fold-enrichment signal across eight cell types and processed by stratifying into four genomic bins as described previously ${ }^{24}$ : bin 0 contains regions with below-baseline signal (fold enrichment versus input $<1$ ), while bins $1-3$ are approximately equal-sized bins that cover the remainder of the genome. RNA-seq data from Roadmap were processed by averaging across eight cell types; bin 0 contains non-expressed genes $(\mathrm{FPKM}=0)$ and intergenic DNA not listed as expressed, while bins 1-3 included genes with up to $0.59,5.68$ and above $5.68 \mathrm{FPKM}$, respectively. Replication time was averaged across the eight ENCODE cell types and divided into four equal-sized genomic bins, where bin 0 is latest and bin 3 is earliest replicating Essential genes were estimated from CRISPR screens in cell lines ${ }^{52}$. All enrichment scores shown compare bins 1-3 for a particular feature (replication time, histone marks, gene expression, L1 motif) versus bin 0 of the same feature. Bin 0 therefore always has $\log$ enrichment $=0$ by definition and is not shown on plots. The analyses were restricted to regions of the genome with perfect CRG75 alignability.

Impact of retrotransposition insertions on gene expression. To study the transcriptional impact of a somatic L1 insertion within COSMIC cancer genes and promoters, we used RNA-seq data to compare gene-expression levels in samples with and without somatic L1 insertion. For each somatic L1 insertion within a cancer gene or promoter, we compared the gene FPKM between the sample having the insertion (study sample) and the remaining samples of the same tumor type (control samples). Using the distribution of gene-expression levels in control samples, we calculated the normalized gene expression differences using a Student's $t$-test. To overcome the problems due to multiple testing, false discovery rate-adjusted $P$ values ( $q$ values) were calculated using the Benjamini-Hochberg procedure, and adjusted $P<0.1$ was considered to be significant.

Analysis of processed pseudogene expression. We analyzed the PCAWG RNA-seq data to identify and characterize the transcriptional consequences of somatic integrations of processed pseudogenes (PSD). We interrogated RNA-seq split reads and discordant read pairs, looking for chimeric retrocopies that involved PSDs and target genomic regions. For each PSD insertion somatic call, we extracted all of the RNA-seq reads (when available), mapping the source gene and the insertion target region, together with the RNA-seq unmapped reads for the corresponding sample. Then, we used these reads as a query in BLASTn ${ }^{53} \mathrm{v} \cdot 2.7 .1$ searches against a database that contained all isoforms of the source gene described in RefSeq ${ }^{54}$, together with the genomic sequence ranging from $-5 \mathrm{~kb}$ to $+5 \mathrm{~kb}$ around the PSD integration site. Finally, we looked for RNA-seq discordant read pairs and/ or RNA-seq clipped reads that supported the joint expression of PSD and target site. Only read pairs with one of the mates aligned to the host gene mRNA with $>98 \%$ identity were considered. All expression signals were confirmed by visual inspection with Integrative Genomics Viewer v.2.4.10.

Validation of somatic retrotransposition algorithms. In silico validation of TraFiC-mem. To evaluate the precision and recall of our algorithm TraFiC-mem, we reanalyzed a mock cancer genome into which we had previously seeded known somatic retrotransposition events at different levels of tumor clonality?
To create the artificial, tumoral genome, 10,000 L1 insertion breakpointsincluding solo-L1, partnered and orphan transductions-were randomly distributed in the standard reference genome using BedTools v.2.25.0, of which 9,227 were inserted out of un-sequenced gaps. Then, ART ${ }^{55}$ (v.MountRainier-2016-06-05) was used to generate paired-end read sequencing data for both the standard and the artificial reference genomes to a $38 \times$ coverage. The simulation FASTQ files were aligned into the standard reference genome with BWA-mem ${ }^{56 \mathrm{v} .7 .17}$. Reads from the normal and tumor BAM files were randomly subsampled and merged with samtools v.1.7 at three distinct proportions to also produce tumor samples with $25 \%, 50 \%$ and $75 \%$ clonalities. After that, the four possible tumor and matched normal pairs were processed with TraFiC-mem to call MEIs. For each clonality, the identified MEIs were compared with the list of simulated MEIs to compute the number of true-positive (TP), false-positive (FP), true-negative $(\mathrm{TN})$ and false-negative $(\mathrm{FN})$ calls. Finally, precision and recall were computed as follows: Precision = TP/(TP + FP); Recall = TP/(TP + FN $)$

Validation of TraFiC-mem calls using single-molecule sequencing. We performed validation of 308 putative somatic retrotranspositions identified with TraFiC-mem in one cancer cell line (NCI-H2087) with high retrotransposition rate, and absent in the matched normal cell line (NCI-BL2087) derived from blood, by single-molecule sequencing using Oxford Nanopore technology. Genomic DNA was sheared to $10-\mathrm{kb}$ fragments using Covaris g-TUBEs, and cleaned with $0.4 \times$ Ampure XP Beads. After end-repairing and dA-tailing using the NEBNext End Repair/dA-tailing module (NEB), whole-genome libraries were constructed with the Oxford Nanopore Sequencing 1D ligation library prep kit (SQK-LSK108, Oxford Nanopore Technologies). Genomic libraries were loaded on MinION R9.4 flowcells. We used the Oxford Nanopore basecaller Albacore v.2.0.1 to generate fastq files. After quality filtering of the fastq files and read trimming of the data with Porechop v.0.2.3, we used minimap2 (ref. ${ }^{57}$ ) v.2.10-r764-dirty to map sequencing reads onto the hs $37 \mathrm{~d} 5$ reference genome. Sequencing coverages were $8.2 \times$ (NCI-BL2087) and 9.17× (NCI-H2087), and average read sizes of mapped reads were $\sim 4.5 \mathrm{~kb}$ (NCI-BL2087) and $\sim 11 \mathrm{~kb}$ (NCI-H2087). After obtaining the whole-genome BAM files for each of the 308 putative somatic retrotransposition calls identified with TraFic-mem, we interrogated the long-read tumor BAM file to find reads that validated the event. MEIs supported by at least one Nanopore read in the tumor and absent in the matched normal sample were considered true-positive somatic events, while MEIs not supported by long reads in the tumor and/or present in the matched normal were considered false-positive calls. Overall, we found $4.22 \%(13 / 308)$ false-positive events. False discovery rate (FDR) was estimated as follows: $\mathrm{FDR}=\mathrm{FP} /(\mathrm{TP}+\mathrm{FP})$.

Validation of L1-mediated rearrangements with PCR and single-molecule sequencing. We performed validation of 20 somatic L1-mediated rearrangements, mostly deletions, identified in two cancer cell lines with high retrotransposition rates (NCI-H2009 and NCI-H2087). We carried out PCR followed by single-molecule sequencing of amplicons from the two tumor cell lines and their matched normal samples (NCI-BL2009 and NCI-BL2087) using a MinION from Oxford Nanopore. PCR primers were designed to amplify three regions from each event (namely, $5^{\prime}$-extreme, $3^{\prime}$-extreme and target sites) as shown in Supplementary Fig. 10.

Validation of L1-mediated rearrangements using mate pairs. To further validate and characterize L1-mediated rearrangements, we performed $10 \times$ mate-pair whole-genome sequencing using libraries with two different insert sizes ( $4 \mathrm{~kb}$ and $10 \mathrm{~kb}$ ), which can span the integrated L1 element that caused the deletion, enabling the validation of the involvement of L1 in the generation of such rearrangements. Mate-pair reads (100 nucleotides long) were aligned to the human reference with BWA-mem v.0.7.17. Then, for each candidate L1-mediated rearrangement, we searched for discordant mate-pair clusters that spanned the breakpoints and supported the L1-mediated event. Each event was confirmed by visual inspection of the BAM files using Integrative Genomics Viewer v.2.4.10.

Reporting Summary. Further information on research design is available in the Nature Research Reporting Summary linked to this article.

\section{Data availability}

Somatic and germline variant calls, mutational signatures, subclonal reconstructions, transcript abundance, splice calls and other core data generated by the ICGC/TCGA PCAWG Consortium are available for download at https:// dcc.icgc.org/releases/PCAWG. Additional information on accessing the data, including raw read files, can be found at https://docs.icgc.org/pcawg/data/. In accordance with the data access policies of the ICGC and TCGA projects, most molecular, clinical and specimen data are in an open tier, which does not require access approval. To access potentially identifying information, such as germline alleles and underlying sequencing data, researchers will need to apply to the TCGA Data Access Committee (DAC) via dbGaP (https://dbgap.ncbi.nlm.nih. gov/aa/wga.cgi? page=login) for access to the TCGA portion of the dataset, and to the ICGC Data Access Compliance Office (DACO; http://icgc.org/daco) for the ICGC portion. In addition, to access somatic SNVs derived from TCGA donors, researchers will also need to obtain $\mathrm{dbGaP}$ authorization. 
In addition, the analyses in this paper used a number of datasets that were derived from the raw sequencing data and variant calls (Supplementary Table 8). The individual datasets are available at Synapse (https://www.synapse.org/), which are also mirrored at DCC portal (https://dcc.icgc.org). Full links, filenames, accession numbers and descriptions are detailed in Supplementary Table 8. VCF files for somatic mobile element insertions described specifically in this manuscript can be found at Synapse, under accession number syn21052009, and in DCC portal at https://dcc.icgc.org/releases/PCAWG/retrotransposition.

\section{Code availability}

The core computational pipelines used by the PCAWG Consortium for alignment, quality control and variant calling are available to the public at https://dockstore. org/search?search=pcawg under a GNU General Public License v.3.0, which allows for reuse and distribution. The algorithm for the identification of somatic retrotransposition events (TraFiC-mem) is available at https://gitlab.com/ mobilegenomesgroup/TraFiC (v.1.2.0).

\section{References}

44. Li, H. \& Durbin, R. Fast and accurate long-read alignment with BurrowsWheeler transform. Bioinformatics 26, 589-595 (2010).

45. Dobin, A. et al. STAR: ultrafast universal RNA-seq aligner Bioinformatics 29, 15-21 (2013).

46. Kim, D. et al. TopHat2: accurate alignment of transcriptomes in the presence of insertions, deletions and gene fusions. Genome Biol. 14, R36 (2013).

47. Anders, S., Pyl, P. T. \& Huber, W. HTSeq-a Python framework to work with high-throughput sequencing data. Bioinformatics 31, 166-169 (2015).

48. PCAWG Transcriptome Core Group et al. Genomic basis for RNA alterations in cancer. Nature https://doi.org/10.1038/s41586-020-1970-0 (2020).

49. Gerstung, M. et al. The evolutionary history of 2,658 cancers. Nature https://doi.org/10.1038/s41586-019-1907-7 (2020).

50. Nik-Zainal, S. et al. The life history of 21 breast cancers. Cell 149, 994-1007 (2012).

51. Rheinbay, E. et al. Analyses of non-coding somatic drivers in 2,693 cancer whole genomes. Nature https://doi.org/10.1038/s41586-020-1965-x (2020).

52. Meyers, R. M. et al. Computational correction of copy number effect improves specificity of CRISPR-Cas9 essentiality screens in cancer cells. Nat. Genet. 49, 1779-1784 (2017).

53. Altschul, S. F., Gish, W., Miller, W., Myers, E. W. \& Lipman, D. J. Basic local alignment search tool. J. Mol. Biol. 215, 403-410 (1990).

54. O'Leary, N.A. et al. Reference sequence (RefSeq) database at NCBI: current status, taxonomic expansion, and functional annotation. Nucleic Acids Res. 44, D733-D745 (2016)

55. Huang, W., Li, L., Myers, J. R. \& Marth, G. T. ART: a next-generation sequencing read simulator. Bioinformatics 28, 593-594 (2012).

56. Li, H. \& Durbin, R. Fast and accurate short read alignment with BurrowsWheeler transform. Bioinformatics 25, 1754-1760 (2009).

57. Li, H. Minimap2: pairwise alignment for nucleotide sequences. Bioinformatics 34, 3094-3100 (2018).

\section{Acknowledgements}

J.M.C.T. is supported by European Research Council (ERC) Starting Grant 716290 'SCUBA CANCERS', Ramon y Cajal grant RYC-2014-14999 and Spanish Ministry of Economy, Industry and Competitiveness (MINECO) grant SAF2015-66368-P. B.R.-M., E.G.A., M.S.G. and S.Z. are supported by PhD fellowships from Xunta de Galicia (Spain) ED481A-2016/151, ED481A-2017/299, ED481A-2017/306 and ED481A-2018/199, respectively. F.S. was supported by ERC Starting Grant 757700 'HYPER-INSIGHT', MINECO grant BFU2017-89833-P 'RegioMut', and further acknowledges institutional funding from the MINECO Severo Ochoa award and from the CERCA Programme of the Catalan Government. Y.S.J. was supported by Korea Health Technology R\&D Project through the Korea Health Industry Development Institute (KHIDI), funded by the Ministry of Health \& Welfare, Republic of Korea (grant number HI16C2387).
A.L.B. is supported by MINECO PhD fellowship BES-2016-078166. M.T. was supported by MINECO grant SAF2015-73916-JIN. R.B. received funding through the National Institutes of Health (U24CA210978 and R01CA188228). M.G.B. received funding through MINECO, AEI, Xunta de Galicia and FEDER (BFU2013-41554-P, BFU2016 78121-P, ED431F 2016/019). N.B. is supported by a My First AIRC grant from the Associazione Italiana Ricerca sul Cancro (number 17658). J.D. is a postdoctoral fellow of the Research Foundation Flanders (FWO) and the European Union's Horizon 2020 research and innovation program (Marie Skłodowska-Curie grant agreement number 703594-DECODE). K.C. and Z.C. are supported by NIH R01 CA172652 and U41 HG007497. Z.C. is supported by an American Heart Association Institutional Data Fellowship Award (17IF33890015). P.A.E. is supported by Cancer Research UK. E.A.L. is supported by K01AG051791. I.M. is supported by Cancer Research UK (C57387/ A21777). F.M. is supported by A.I.L. (Associazione Italiana Contro le Leucemie-Linfomi e Mieloma ONLUS) and by S.I.E.S. (Società Italiana di Ematologia Sperimentale). S.M.W received funding through a SNSF Early Postdoc Mobility fellowship (P2ELP3_155365) and an EMBO Long-Term Fellowship (ALTF 755-2014). J.W. received funding from the Danish Medical Research Council (DFF-4183-00233). D.C.W. is funded by the Li Ka Shing foundation and the National Institute for Health Research (NIHR) Oxford Biomedical Research Centre. J.O.K. is supported by an ERC Starting Grant. P.V.L. is a Winton Group Leader in recognition of the Winton Charitable Foundation's support towards the establishment of The Francis Crick Institute. This work is supported by The Francis Crick Institute, which receives its core funding from Cancer Research UK (FC001202), the UK Medical Research Council (FC001202) and the Wellcome Trust (FC001202). H.H.K. is supported by grants from the National Institute of General Medical Sciences (P50GM107632 and 1R01GM099875). K.H.B. is supported by P50GM107632, R01CA163705 and R01GM124531. This work was supported by the TransTumVar project PN013600. R.C.F. thanks Cancer Research UK Programme Grant for esophageal ICGC, Cambridge BRC and ECMC infrastructure support. This work was supported by the Wellcome Trust grant 09805 . We acknowledge the contributions of the many clinical networks across the ICGC and TCGA who provided samples and data to the PCAWG Consortium, and the contributions of the Technical Working Group and the Germline Working Group of the PCAWG Consortium for collation, realignment and harmonized variant calling of the cancer genomes used in this study. We thank the patients and their families for their participation in the individual ICGC and TCGA projects.

\section{Author contributions}

J.M.C.T. and P.J.C. designed the study and wrote the manuscript with assistance from B.R.-M., H.H.K. and K.H.B. B.R.-M., A.B.-O., J.Z. and J.M.C.T. designed the somatic retrotransposition algorithms and performed retrotransposition analyses with support from A.L.B., E.G.A., S.Z., Z.C., K.C., E.A.L., D.A., J.M., G.B., M.G.B., H.D., F.C.P.N., S.M.W., M.G., P.A.E., P.J.P., H.H.K. and K.H.B. F.S. carried out genomic features analyses and wrote the associated manuscript section. Y.S.J. performed transcriptomic analyses. A.D.-B., M.P., B.R.-M., D.T. and J.M.C.T. performed the analysis of processed pseudogenes. J.D., A.B.-O., S.C.D. and P.V.L. carried out copy-number analyses. Y.L., N.D.R., S.E.S., J.W., J.A.W., R.B., J.O.K. and P.J.C. performed structural variation analyses. B.R.-M., J.Z., I.M., D.C.W. and P.V.L. carried out statistical analyses. J.T., J.R.-C., M.T., M.S. and D.G.-S. performed sample preparation, experimental validation and evaluation of the sequencing data. K.M.R., J.Z. and A.B. supported variant calling algorithms and sequencing analysis platforms. F.M., N.B., G.C. and R.C.F. provided additional genomic data, samples and experimental materials. All authors reviewed the manuscript during its preparation. R.B. and P.J.C. were working group or project co-leaders.

\section{Competing interests}

The authors declare no competing interests.

\section{Additional information}

Supplementary information is available for this paper at https://doi.org/10.1038/ s41588-019-0562-0.

Correspondence and requests for materials should be addressed to P.J.C. or J.M.C.T.

Reprints and permissions information is available at www.nature.com/reprints. 


\section{natureresearch}

\section{Reporting Summary}

Nature Research wishes to improve the reproducibility of the work that we publish. This form provides structure for consistency and transparency in reporting. For further information on Nature Research policies, see Authors \& Referees and the Editorial Policy Checklist.

\section{Statistics}

For all statistical analyses, confirm that the following items are present in the figure legend, table legend, main text, or Methods section.

n/a Confirmed

$\bigotimes$ The exact sample size $(n)$ for each experimental group/condition, given as a discrete number and unit of measurement

$\bigotimes$ A statement on whether measurements were taken from distinct samples or whether the same sample was measured repeatedly

The statistical test(s) used AND whether they are one- or two-sided

Only common tests should be described solely by name; describe more complex techniques in the Methods section.

$\bigotimes$ A description of all covariates tested

A description of any assumptions or corrections, such as tests of normality and adjustment for multiple comparisons

A full description of the statistical parameters including central tendency (e.g. means) or other basic estimates (e.g. regression coefficient)

AND variation (e.g. standard deviation) or associated estimates of uncertainty (e.g. confidence intervals)

For null hypothesis testing, the test statistic (e.g. $F, t, r$ ) with confidence intervals, effect sizes, degrees of freedom and $P$ value noted

Give $P$ values as exact values whenever suitable.

For Bayesian analysis, information on the choice of priors and Markov chain Monte Carlo settings

For hierarchical and complex designs, identification of the appropriate level for tests and full reporting of outcomes

Estimates of effect sizes (e.g. Cohen's d, Pearson's r), indicating how they were calculated

Our web collection on statistics for biologists contains articles on many of the points above.

\section{Software and code}

Policy information about availability of computer code

Data collection

Data and metadata were collected from International Cancer Genome Consortium (ICGC) consortium members using custom software packages designed by the ICGC Data Coordinating Centre. The general-purpose core libraries and utilities underlying this software have been released under the GPLv3 open source license as the "Overture" package and are available at https://www.overture.bio. Other data collection software used in this effort, such as ICGC-specific portal user interfaces, are available upon request to contact@overture.bio.

Data analysis Somatic mobile element insertions (MEIs) were identified with TraFiC-mem v1.2.0 (https://gitlab.com/mobilegenomesgroup/TraFiC). MEls target genomic regions were annotated using ANNOVAR v2016-02-01 and GENCODE v19 annotation. Oxford Nanopore Technologies (ONT) sequencing data was generated and processed using these packages: MinKNOW v18.01.6; Albacore v2.0.1; Porechop v0.2.3; minimap2 v2.10-r764-dirty74; Samtools v1.7. Simulated data containing MEls were realigned using biobambam v2.0.25 and BWAmem v0.7.17.

The workflows executing core WGS alignment, QC and variant-calling software are packaged as executable Dockstore images and available at: https://dockstore.org/search?labels.value.keyword=pcawg\&searchMode=files. Individual software components are as follows: BWA-MEM v0.78.8-r455; DELLY v0.6.6; ACEseq v1.0.189; DKFZ somatic SNV workflow v1.0.132-1; Platypus v0.7.4; ascatNgs v1.5.2; BRASS v4.012; grass v1.1.6; CaVEMan v1.50; Pindel v1.5.7; ABSOLUTE/JaBbA v1.5; SvABA 2015-05-20; dRanger 2016-03-13; BreakPointer 2015-12-22; MuTect v1.1.4; MuSE v1.Orc; SMuFIN 2014-10-26; OxoG 2016-4-28; VAGrENT v2.1.2; ANNOVAR V2014Nov12; VariantBAM V2017Dec12; SNV-Merge v2017May26; SV-MERGE V2017Dec12; DKFZ V2016Dec15. 
Policy information about availability of data

All manuscripts must include a data availability statement. This statement should provide the following information, where applicable:

- Accession codes, unique identifiers, or web links for publicly available datasets

- A list of figures that have associated raw data

- A description of any restrictions on data availability

Somatic mobile element insertion calls can be found in Synapse at https://www.synapse.org/ with accession code syn21052009 and in DCC Portal at https:// dcc.icgc.org/releases/PCAWG/retrotransposition

WGS somatic and germline variant calls, mutational signatures, subclonal reconstructions, transcript abundance, splice calls and other core data generated by the ICGC/TCGA Pan-cancer Analysis of Whole Genomes Consortium are available for download at https://dcc.icgc.org/releases/PCAWG. Additional information on accessing the data, including raw read files, can be found at https://docs.icgc.org/pcawg/data/. In accordance with the data access policies of the ICGC and TCGA projects, most molecular, clinical and specimen data are in an open tier which does not require access approval. To access potentially identification information, such as germline alleles and underlying sequencing data, researchers will need to apply to the TCGA Data Access Committee (DAC) via dbGaP (https:// dbgap.ncbi.nlm.nih.gov/aa/wga.cgi?page=login) for access to the TCGA portion of the dataset, and to the ICGC Data Access Compliance Office (DACO; http:// icgc.org/daco) for the ICGC portion. In addition, to access somatic single nucleotide variants derived from TCGA donors, researchers will also need to obtain dbGaP authorization

\section{Field-specific reporting}

Please select the one below that is the best fit for your research. If you are not sure, read the appropriate sections before making your selection. $\bigotimes$ Life sciences Behavioural \& social sciences Ecological, evolutionary \& environmental sciences

For a reference copy of the document with all sections, see nature.com/documents/nr-reporting-summary-flat.pdf

\section{Life sciences study design}

All studies must disclose on these points even when the disclosure is negative.

Sample size

We compiled an inventory of matched tumour/normal whole cancer genomes in the ICGC Data Coordinating Centre. Most samples came from treatment-naïve, primary cancers, but there were a small number of donors with multiple samples of primary, metastatic and/or recurrent tumours. Our inclusion criteria were: (i) matched tumour and normal specimen pair; (ii) a minimal set of clinical fields; and (iii) characterisation of tumour and normal whole genomes using Illumina HiSeq paired-end sequencing reads.

We collected genome data from 2,834 donors, representing all ICGC and TCGA donors that met these criteria at the time of the final data freeze in autumn 2014.

Data exclusions Based on the robustness of the retrotransposition calls (false discovery rate $<5 \%$ ), we opted to retain all samples preliminarly excluded by the PCAWG Consortium, as they were excluded from single-nucleotide variants and structural variation analyses based on read direction biases from PCR artifacts or poor sequence quality, but were not found to be problematic for retrotransposition analysis.

Replication The core pipeline for the identification of somatic mobile element insertions, TraFic-mem, was validated through long-read sequencing data analysis in one cancer cell-line with high retrotransposition rate and its matched normal sample. 295 out of 308 somatic retrotransposition events were confirmed through the long-reads (false discovery rate $<5 \%$ ). In addition, TraFiC-mem was assessed using simulated data containing MEls at different levels of tumour clonality. This analysis confirmed a high precision (>99\%) and a recall ranging from 90 to $94 \%$ for tumour clonalities from 25 to 100\%, respectively.

In order to evaluate the performance of each of the mutation-calling pipelines and determine an integration strategy, we performed a largescale deep sequencing validation experiment. We selected a pilot set of 63 representative tumour/normal pairs, on which we ran the three core pipelines, together with a set of 10 additional somatic variant-calling pipelines contributed by members of the SNV Calling Working Group. Overall, the sensitivity and precision of the consensus somatic variant calls were 95\% (C190\%: 88-98\%) and 95\% (C190\%: 71-99\%) respectively for SNVs. For somatic indels, sensitivity and precision were 60\% (34-72\%) and 91\% (73-96\%) respectively. Regarding SVs, we estimate the sensitivity of the merging algorithm to be $90 \%$ for true calls generated by any one caller; precision was estimated as $97.5 \%$ - that is, $97.5 \%$ of SVs in the merged SV call-set have an associated copy number change or balanced partner rearrangement.

Randomization No randomisation was performed.

Blinding

No blinding was undertaken.

\section{Reporting for specific materials, systems and methods}

We require information from authors about some types of materials, experimental systems and methods used in many studies. Here, indicate whether each material, system or method listed is relevant to your study. If you are not sure if a list item applies to your research, read the appropriate section before selecting a response. 


\begin{tabular}{l|l}
\hline $\mathrm{n} / \mathrm{a}$ & Involved in the study \\
\hline & $\square$ Antibodies \\
$\triangle$ & $\square$ Eukaryotic cell lines \\
$\square$ & $\square$ Palaeontology \\
$\square$ & $\square$ Animals and other organisms \\
$\square$ & $\bigotimes$ Human research participants \\
$\triangle$ & $\square$ Clinical data
\end{tabular}

\section{Human research participants}

Policy information about studies involving human research participants

Population characteristics

Recruitment

Ethics oversight
Patient-by-patient clinical data are provided in the marker paper for the PCAWG consortium (Extended Data Table 1 of that manuscript). Demographically, the cohort included 1,469 males (55\%) and 1,189 females (45\%), with a mean age of 56 years (range, 1-90 years). Using population ancestry-differentiated single nucleotide polymorphisms (SNPs), the ancestry distribution was heavily weighted towards donors of European descent (77\% of total) followed by East Asians (16\%), as expected for large contributions from European, North American and Australian projects. We consolidated histopathology descriptions of the tumour samples, using the ICD-0-3 tumour site controlled vocabulary. Overall, the PCAWG data set comprises 38 distinct tumour types. While the most common tumour types are included in the dataset, their distribution does not match the relative population incidences, largely due to differences among contributing ICGC/TCGA groups in numbers sequenced.

Patients were recruited by the participating centres following local protocols.

The Ethics oversight for the PCAWG protocol was undertaken by the TCGA Program Office and the Ethics and Governance Committee of the ICGC. Each individual ICGC and TCGA project that contributed data to PCAWG had their own local arrangements for ethics oversight and regulatory alignment. 